\title{
LUTTINGER MODEL AND LUTTINGER LIQUIDS
}

\author{
Vieri Mastropietro \\ University of Rome Tor Vergata, Math. Dept \\ Viale della Ricerca Scientifica 00133, Rome, Italy
}

\begin{abstract}
The Luttinger model owes its solvability to a number of peculiar features, like its linear relativistic dispersion relation, which are absent in more realistic fermionic systems. Nevertheless according to the Luttinger liquid conjecture a number of relations between exponents and other physical quantities, which are valid in the Luttinger model, are believed to be true in a wide class of systems, including tight binding or jellium one dimensional fermionic systems. Recently a rigorous proof of several Luttinger liquid relations in non solvable models has been achieved; it is based on exact Renormalization Group and methods coming from Constructive Quantum Field Theory and its main steps will be reviewed below.
\end{abstract}

\section{$0.1 \quad$ Introduction}

The crucial observation that the low energy excitations of one-dimensional metals are well described in terms of $1+1$ massless Dirac fermions dates back to Tomonaga [1] and it was one of the earliest examples of emerging Quantum Field Theory (QFT) description in condensed matter physics; an idea which, later on, found several applications including the recent one to graphene (which is described by Dirac fermions in $d=2+1$ ). The QFT description of 1D metals in [1] is based on several unproven assumptions and, in order to separate approximations from exact results, Luttinger [2] proposed a model, nowadays called Luttinger model, incorporating the Tomonaga approximations in it; the quasi-particle excitations are described in terms of two kinds of spinless fermions with linear dispersion relation, and a Dirac sea is introduced filling the infinite states with negative energy. The Luttinger model looks quite similar to the Thirring model [3] proposed a little earlier, as it was noted by Luttinger himself; both models describe massless Dirac fermions in $d=1+1$ with an interaction quartic in the fermionic fields. There are however important differences: while in the Thirring model the quartic current-current interaction is local, the 
Luttinger interaction is short ranged; moreover the ultraviolet cut-off (to be removed) is only on the momenta and not on the energies. As a consequence, the Thirring model is plagued by ultraviolet divergences and is Lorentz invariant, while the Luttinger model has no ultraviolet divergences and Lorentz invariance is lost.

Luttinger correctly observed that his model should be solvable, but unfortunately the solution he proposed was not correct. Curiously, the fate of the Luttinger model was then somewhat similar to the one of the Thirring model, whose exact solution was anticipated by several uncorrect attempts, including the one of Thirring himself; the solution of the Thirring model was finally found by Johnson [4] assuming the validity of anomalous commutators as functions of two parameters which are fixed by a selfconsistence argument.

The solution of the Luttinger model was found by Mattis and Lieb [5] showing that the density operators verify anomalous commutation relations, a crucial point which was missed by Luttinger, and showing that the fermionic Luttinger hamiltonian can be mapped in a quadratic boson hamiltonian; this allows the determination of all the correlations functions and of several physical quantities. It was found that the interaction radically alters the physical behavior. The occupation number, which has a discontinuity in the absence of interaction, becomes continuous and the wave function renormalization diverges at the Fermi points with a critical exponent non trivial function of the coupling. By the solution in [5] one can compute the $n$-point functions and the response functions, whose large distance behavior have also anomalous exponents.

The exact determination of all its correlations makes the Luttinger model rather unique with respect to other solvable fermionic models, like the Hubbard model, in which only the ground state energy or the energy of some excited states can be computed. On the other hand, the Luttinger model has been proposed as a caricature of a true many body fermionic hamiltonian, and owes its exact solvability to certain peculiar features (for instance the linear dispersion relation and the introduction of a Dirac sea) which are surely unrealistic. It is then natural to ask which features of the Luttinger model are still true in a realistic hamiltonian.

It is reasonable to expect, according to [1], that anomalous critical exponents are generically present (at least in the absence of the spin) in 1D metals. The exponents will depend on the model details and of course differ from the Luttinger model ones; moreover for generic models there is in general no exact solution, or even if it is (as by Bethe ansatz in the 
$X X Z$ chain) only certain eigenvalues of the energy can be computed and not the exponents. It is then clear the importance of the Luttinger liquid conjecture proposed by Haldane [6], [7] (see also [8]), according to which a number of exact simple relations between exponents and other physical quantities, which are valid in the Luttinger model, are still true in a wide class of more realistic one dimensional models. In particular, according to such conjecture, one can determine all the exponents in terms of a single one, or in terms of (say) the Fermi velocity and the susceptibility.

The validity of the Luttinger liquid conjecture is a non trivial statement, connected with the extended scaling relations proposed by Kadanoff [9] for two dimensional classic statistical mechanics models like the Eight vertex or the Ashkin-Teller model. A justification of the Luttinger liquid conjecture is sometimes considered the fact that, according to a Renormalization Group (RG) analysis, interacting 1D fermionic systems with non linear dispersion relation differ from the Luttinger model through irrelevant terms in the RG sense. However this simple argument is not correct; the irrelevant terms change the exponents and break a number of symmetries which are instead true in the Luttinger model and which are essential for its solvability. Therefore, one has to prove that the symmetry-breaking effects due to the lattice or non linear bands, while changing the exponents, do not change the relations between exponents and other physical parameters. Usual approximate RG methods neglect the irrelevant terms so that they are not suitable to face this problem. In recent years the methods developed in constructive Quantum Field Theory have been applied to many body theory. Such methods are exact (that is the irrelevant terms are taken into account) and rigorous, as the physical observables can be written in terms of renormalized expansion, whose convergence can be proved at least if the coupling is not too large. Using this approach several Luttinger liquid relations in a wide class of models in generic fermionic one dimensional models has been recently proven, and here we will review the main ideas behind such proof. In the second section we recall the Luttinger model and its solution. In the third section we introduce a generic model for 1D fermions on a lattice and we recall the Luttinger liquid conjecture. In the fourth section we review the exact Renormalization Group analysis of a generic (non solvable) 1D lattice fermionic system, leading to a convergent expansion of the physical obervables in terms of running coupling constants. In the fifth section we will show that such running coupling constants do not increase under RG iterations as consequence of Ward Identities. In order to do that, one has to face and solve a well known problem, which is present in any Wilso- 
nian RG approach; the momentum cut-off breaks the local symmetries and produces additional terms in the Ward identities which must be taken into account. In the sixth section we will derive emerging Ward Identities for the lattice model related to the validity of asymptotic symmetries (broken by irrelevant terms) which will be used in the seventh section for proving the Luttinger liquid relations for non solvable lattice models.

\subsection{The Luttinger model exact solution}

The Luttinger model hamiltonian is

$$
\begin{aligned}
& H=H_{0}+V=\int_{0}^{L} d x \sum_{\varepsilon= \pm}: \widetilde{\psi}_{x, \varepsilon}^{+} \varepsilon \partial \widetilde{\psi}_{x, \varepsilon}^{-}:+ \\
& \lambda \int d x d y v(x-y)\left[\sum_{\varepsilon= \pm} q_{1, \varepsilon}: \widetilde{\psi}_{\vec{x}, \varepsilon}^{+} \widetilde{\psi}_{\vec{x}, \varepsilon}^{-}:\right]\left[\sum_{\varepsilon= \pm} q_{2, \varepsilon}: \widetilde{\psi}_{\vec{y}, \varepsilon}^{+} \widetilde{\psi}_{\vec{y}, \varepsilon}^{-}:\right]
\end{aligned}
$$

where if $\varepsilon= \pm$

$$
\psi_{x, \varepsilon}^{ \pm}=e^{ \pm i \varepsilon p_{F} x} \widetilde{\psi}_{x, \varepsilon}^{ \pm}=\frac{e^{ \pm i \varepsilon p_{F} x}}{\sqrt{L}} \sum_{k} e^{ \pm i k x} \widehat{a}_{k, \varepsilon}^{ \pm}
$$

and $\widetilde{\psi}_{x, \varepsilon}^{ \pm}$is a fermionic field with periodic boundary conditions, $p_{F}$ is the Fermi momentum, $v(x-y)$ is a short range potential $|\widehat{v}(p)| \leq C e^{-\zeta|p|}$ with $\zeta>0$ a constant, and the Wick ordering is defined rearranging the order so that $a_{-k,+}^{+}, a_{k,+}^{-}, a_{k,-}^{+}, a_{-k,-}^{-}, k \geq 0$ are always to the right of the other operators, and the new product is multiplied by the parity sign necessary to produce it. Finally $q_{i, \varepsilon}$ are charges, and for definiteness we will follow the original choice of $[2]$

$$
q_{1,+}=q_{2,-}=1
$$

and zero otherwise; this choice is done only for definiteness and the model is solvable for a wide class of charges $q_{i, \varepsilon}$. With this choice of the charges the Hamiltonian can be rewritten as

$$
\begin{aligned}
& H_{0}=\sum_{\varepsilon, k>0} k\left(\widehat{a}_{\varepsilon k, \varepsilon}^{+} \widehat{a}_{\varepsilon k, \varepsilon}^{-}+\widehat{a}_{-\varepsilon k, \varepsilon}^{-} \widehat{a}_{-\varepsilon k, \varepsilon}^{+}\right) \\
& V=\frac{\lambda}{L} \sum_{p>0} \widehat{v}(p)\left[\rho_{+}(p) \rho_{-}(-p)+\rho_{+}(-p) \rho_{-}(p)\right]+\frac{\lambda \widehat{v}(0)}{L} N_{1} N_{-1}
\end{aligned}
$$

where

$$
\rho_{\varepsilon}(p)=\sum_{k} \widehat{a}_{k+p, \varepsilon}^{+} \widehat{a}_{k, \varepsilon} \quad N_{\varepsilon}=\sum_{k>0}\left(\widehat{a}_{\varepsilon k, \varepsilon}^{+} \widehat{a}_{\varepsilon k, \varepsilon}^{-}-\widehat{a}_{-\varepsilon k, \varepsilon}^{-} \widehat{a}_{-\varepsilon k, \varepsilon}^{+}\right)
$$


The regularization which is implicit in the above Hamiltonian is the suppression of the modes $|k| \geq \Lambda$ for the fermion with momentum $k$, where $\Lambda$ is a momentum cut-off to be removed $\Lambda \rightarrow \infty$; that is $\rho_{\varepsilon}(p)$ has be thought as $\sum_{k} \chi_{\Lambda}(k+p) \chi_{\Lambda}(k) \widehat{a}_{k+p, \varepsilon}^{+} \widehat{a}_{k, \varepsilon}^{-}$where $\chi_{\Lambda}(k)=1$ for $|k| \leq \Lambda$ and zero otherwise.

The Hamiltonian (0.2) can be regarded as an operator defined on the Hilbert space $\mathcal{H}$ constructed as follows. If $\mid 0>$ is a state (the "Dirac sea" state) such that $\widehat{a}_{k,-}^{+} \mid 0>=0$ and $\widehat{a}_{-k,+}^{+} \mid 0>=0$ for $k \geq 0, \mathcal{H}_{0}$ is the linear span of all the states obtained applying finitely many creation or annihilation operators on $\mid 0>$ and $\mathcal{H}$ is the completion of $\mathcal{H}_{0}$. Note that $H$ is a bounded operators over $\mathcal{H}$ provided that $\widehat{v}(p)$ verifies the stability condition $|\lambda \widehat{v}(p)| \leq 2 \pi$. The crucial observation of Mattis and Lieb was that the density operators verify the following anomalous commutation relations

$$
\left[\rho_{\varepsilon}(-p), \rho_{\varepsilon}\left(p^{\prime}\right)\right]=\varepsilon \frac{p L}{2 \pi} \delta_{p, p^{\prime}} \quad p>0
$$

This follows noting that the commutator when $p=p^{\prime}$ is equal to

$$
-\sum_{k=-\Lambda+p}^{\Lambda} \widehat{a}_{k, \varepsilon}^{+} \widehat{a}_{k, \varepsilon}^{-}+\sum_{k=-\Lambda}^{\Lambda-p} \widehat{a}_{k, \varepsilon}^{+} \widehat{a}_{k, \varepsilon}^{-}=\sum_{k=-\Lambda}^{-\Lambda+p} \widehat{a}_{k, \varepsilon}^{+} \widehat{a}_{k, \varepsilon}^{-}-\sum_{k=\Lambda-p}^{\Lambda} \widehat{a}_{k, \varepsilon}^{+} \widehat{a}_{k, \varepsilon}^{-}
$$

which on any state in $\mathcal{H}$ is, in the limit $\Lambda \rightarrow \infty$, equal to $\varepsilon \frac{p L}{2 \pi}$. Other important relations are, for $p>0$

$$
\left[H_{0}, \rho_{\varepsilon}(p)\right]=-\varepsilon p \varepsilon_{\varepsilon}(p) \quad\left[\rho_{\varepsilon}(p), \sum_{\varepsilon, p>0} \rho_{\varepsilon}(\varepsilon p) \rho_{\varepsilon}(-\varepsilon p)\right]=-\varepsilon p \frac{L}{2 \pi} \rho_{\varepsilon}(p)
$$

and $\rho_{\varepsilon}(-\varepsilon p) \mid 0>=0$. We can write

$$
H=H_{1}+H_{2}
$$

where, if $T=\frac{2 \pi}{L} \sum_{\varepsilon, p>0} \rho_{\varepsilon}(\varepsilon p) \rho_{\varepsilon}(-\varepsilon p)$

$$
\begin{aligned}
& H_{1}=H_{0}-T+\frac{\lambda \widehat{v}(0)}{L} N_{+} N_{-} \\
& H_{2}=T+\frac{\lambda}{L} \sum_{p>0} \widehat{v}(p)\left[\rho_{+}(p) \rho_{+}(-p)+\rho_{-}(-p) \rho_{-}(p)\right]
\end{aligned}
$$

and $\left[H_{1}, \rho_{\varepsilon}( \pm p)\right]=0$ for $p>0$, while $H_{2}$ can be easily diagonalized; if $S=\frac{2 \pi}{L} \sum_{p \neq 0} p^{-1} \rho_{+}(p) \rho_{-}(-p)$

$$
e^{i S} H_{2} e^{-i S}=\frac{2 \pi}{L} \sum_{p>0} \varepsilon(p)\left[\rho_{+}(p) \rho_{+}(-p)+\rho_{-}(p) \rho_{-}(-p)\right]+E_{0}
$$


where $E_{0}$ is a constant and

$$
\varepsilon(p)=\operatorname{sech}(2 \phi(p)) \quad \tanh 2 \phi=-\frac{\lambda \widehat{v}(p)}{2 \pi}
$$

The above formula (0.12) refers to the specific choice (0.3) of the $q_{i, \varepsilon}$, but a general formula can be easily obtained. The set of states $\mid j, n_{1}, n_{2}>$ obtained applying operators $\rho_{+}(p), \rho_{-}(-p)$ an arbitrary number of times on the state in which all the levels are filled up to $n_{1}$ with fermions of type + and down to level $n_{2}$ is complete $[10 ; 6]$ and $H_{1}$ is a constant in the subspace with fixed $n_{1}, n_{2}$, from the fact that $\left[H_{1}, \rho_{\varepsilon}( \pm p)\right]=0$. Therefore $e^{-i S} \mid 0>$ is the ground state of $H$ as $H_{1}$ is constant on the subspace with $n_{1}=n_{2}=0$ while

$$
H_{2} e^{-i S}\left|0>=e^{-i S}\left(e^{i S} H_{2} e^{-i S}\right) 0>=E_{0} e^{-i S}\right| 0>
$$

Defining $\widetilde{\psi}_{\mathbf{x}, \varepsilon}=e^{H t} \widetilde{\psi}_{x, \varepsilon} e^{-H t}, \mathbf{x}=(t, x)$ the n-point function is given by

$$
<\widetilde{\psi}_{\mathbf{x}_{1}, \varepsilon_{1}}^{\sigma_{1}} \ldots \widetilde{\psi}_{\mathbf{x}_{n}, \varepsilon_{n}}^{\sigma_{n}}>\equiv<0\left|e^{i S} \mathcal{T}\left(\widetilde{\psi}_{\mathbf{x}_{1}, \varepsilon_{1}}^{\sigma_{1}} \ldots \widetilde{\psi}_{\mathbf{x}_{n}, \varepsilon_{n}}^{\sigma_{n}}\right) e^{-i S}\right| 0>
$$

where $\mathcal{T}$ is the time ordering. In [5] only the case $n=2, t_{1}=t_{2}=0$ was studied, but it is only a matter of algebra to deduce from the exact solution the explicit form of the time-dependent $n$-point correlation; this was done in [11] for the 2-point and in [12], [13] for the $n$-point function. It is found

$$
<\widetilde{\psi}_{\mathbf{x}_{1}, \varepsilon_{1}}^{\sigma_{1}} \ldots \widetilde{\psi}_{\mathbf{x}_{n}, \varepsilon_{n}}^{\sigma_{n}}>=<\widetilde{\psi}_{\mathbf{x}_{1}, \varepsilon_{1}}^{\sigma_{1}} \ldots \widetilde{\psi}_{\mathbf{x}_{n}, \varepsilon_{n}}^{\sigma_{n}}>_{0} e^{-Q_{n}}
$$

where $<\widetilde{\psi}_{\mathbf{x}_{1}, \varepsilon_{1}}^{\sigma_{1}} \ldots \widetilde{\psi}_{\mathbf{x}_{n}, \varepsilon_{n}}^{\sigma_{n}}>_{0}$ is the non-interacting $\lambda=0$ (imaginary time) n-point function (expressed by the Wick rule in terms of the 2-point free function) and

$$
\begin{aligned}
& Q_{n}=\frac{2 \pi}{L} \sum_{p>0} \sum_{\varepsilon= \pm}\left\{s^{2}(p)\left[\frac{n}{2}+\sum_{i, j \in I_{\varepsilon}, i<j} \sigma_{i} \sigma_{j} e^{-p\left|t_{j}-t_{j}\right| \varepsilon(p)} \cos p\left(x_{i}-x_{j}\right)\right]\right. \\
& -c(p) s(p) \sum_{i \in I_{\varepsilon}, j \in I_{-\varepsilon}} \sigma_{i} \sigma_{j} e^{-p\left|t_{j}-t_{j}\right| \varepsilon(p)} \cos p\left(x_{i}-x_{j}\right) \\
& -\sum_{i, j \in I_{\varepsilon}, i<j}\left[e^{-\left|t_{i}-t_{j}\right|}-e^{-p\left|t_{i}-t_{j}\right| \varepsilon(p)}\right] \cos p\left(x_{i}-x_{j}\right) \\
& \left.-i \varepsilon \sum_{i, j \in I_{\varepsilon}, i<j} \sigma_{i} \sigma_{j} \frac{t_{j}-t_{j}}{\left|t_{i}-t_{j}\right|}\left[e^{-\left|t_{i}-t_{j}\right|}-e^{-p\left|t_{i}-t_{j}\right| \varepsilon(p)}\right] \sin p\left(x_{i}-x_{j}\right)\right\}
\end{aligned}
$$

where

$$
s(p)=\sinh \phi(p) \quad c(p)=\cosh \phi(p)
$$


In particular the 2-point function is given by

$$
\begin{aligned}
& <\widetilde{\psi}_{\mathbf{x}, \varepsilon}^{-} \widetilde{\psi}_{0, \varepsilon}^{+}>=\frac{1}{2 \pi} \frac{1}{i \varepsilon x+t} e^{-Q_{2}(\mathbf{x})} \\
& Q_{2}(\mathbf{x})=\int_{0}^{\infty} \frac{d p}{p}\left[2 s^{2}(p)\left(1-e^{-p \varepsilon(p)|t|} \cos p x\right)+\right. \\
& \left.\left(\cos p x-i \varepsilon \frac{t}{|t|} \sin p x\right)\left(e^{-p|t|}-e^{-p \varepsilon(p)|t|}\right)\right]
\end{aligned}
$$

From the above expression is easy to see that the non locality of the two body potential is essential to have a finite 2-point function; if we consider a local potential then $\widehat{v}(p)=1$ and the above expression is diverging. Note also that (0.19) has the same ultraviolet divergence at $\mathbf{x}=\mathbf{0}$ than the free 2 -point function as $Q_{2}(\mathbf{0})=0$. On the contrary the large distance behavior is different with respect to the non interacting case and given by

$$
<\widetilde{\psi}_{\mathbf{x}, \varepsilon}^{-} \widetilde{\psi}_{0, \varepsilon}^{+}>\sim \sim_{|\mathbf{x}| \rightarrow \infty} \frac{1}{i \varepsilon x+v_{F} t} \frac{A(\lambda)}{\left|x^{2}+v_{F}^{2} t^{2}\right|^{\eta / 2}}
$$

with $A(\lambda)$ a suitable constant and

$$
\begin{aligned}
& v_{F}=\varepsilon(0)=\sqrt{1-(\lambda \widehat{v}(0) / 2 \pi)^{2}} \\
& \eta=2 \sinh ^{2} \phi(0)=\frac{1}{2}\left[K+K^{-1}-2\right] \quad K=e^{2 \phi}=\sqrt{\frac{1+\lambda \widehat{v}(0) / 2 \pi}{1-\lambda \widehat{v}(0) / 2 \pi}}
\end{aligned}
$$

From (0.22) we see that the interaction not only modifies the Fermi velocity but also changes qualitatively the asymptotic infrared behavior of the 2-point function, producing an anomalous dimension with exponent $1+\eta$, with $\eta>0$ and non trivial function of the coupling. The presence of anomalous exponents is one of the most interesting feature of the Luttinger model, and implies a rather different physical behavior with respect to the non interacting system; for instance the occupation number is not discontinuous as in the $\lambda=0$ case, but it becomes continuous and such that $n_{k+p_{F}}-n_{p_{F}} \sim|k|^{\eta}$ for small $k$.

It is also important to compare the the 2-point function of the Luttinger model with the 2-point function $S_{t h}(\mathbf{x})$ of the Thirring model, as computed in $[4]$

$$
S_{t h}(\mathbf{x})=\frac{1}{2 \pi} \frac{1}{i \varepsilon x+t} \frac{1}{\left|x^{2}+t^{2}\right|^{\eta}}
$$

Despite the similar long distance behavior, there are important differences; the ultraviolet short distance behavior is different with respect the the free one, and the light velocity is not changed due to Lorentz invariance. 
From (0.15) we can write the response function; if we defined the total density $\rho_{\mathbf{x}}^{C D W}=\lim _{\alpha \rightarrow 0} \sum_{\varepsilon} e^{2 i \varepsilon p_{F} x} \widetilde{\psi}_{x, t+\alpha \varepsilon}^{+} \widetilde{\psi}_{\mathbf{x},-\varepsilon}^{-}$then, using a pointsplitting procedure from (0.15), for $|\mathbf{x}| \rightarrow \infty$, if $T$ denotes truncation

$$
<\rho_{\mathbf{x}}^{C D W} \rho_{0}^{C D W}>_{T} \sim(1+A(\lambda)) \frac{\cos \left(2 p_{F} x\right)}{2 \pi^{2}\left(v_{F}^{2} t^{2}+x^{2}\right)^{X_{+}}}
$$

with $X_{+}$is a critical exponent given by

$$
X_{+}=K
$$

Similarly we can introduce the Cooper pair density

$$
\rho_{\mathbf{x}}^{c}=\lim _{\alpha \rightarrow 0} \sum_{\varepsilon, \sigma} \psi_{x, t+\alpha, \varepsilon}^{\sigma} \psi_{x, x_{0},-\varepsilon}^{\sigma}
$$

and one gets

$$
<\rho_{\mathbf{x}}^{c} \rho_{0}^{c}>_{T} \sim(1+A(\lambda)) \frac{1}{2 \pi^{2}\left(v_{F}^{2} t^{2}+x^{2}\right)^{X_{-}}}
$$

with

$$
X_{-}=K^{-1}
$$

Note that $(0.22),(0.24),(0.27)$ relates the exponents by simple relations; while the exponents depend from the details of the model (0.2) (for instance from the charges $q_{i, \varepsilon}$ ) such relations are true for any choice of the charges for which the solvability holds. For instance if $q_{i, \varepsilon}=\frac{1}{2}$ then $(0.22),(0.24),(0.27)$ are still true even if $K$ has a different expression

$$
K=\sqrt{1+\frac{\lambda \widehat{v}(0)}{2 \pi}}
$$

As we will see in the following section such relations between exponents have been proposed to be true in a wide class of models, even when an exact solution is lacking.

Defining the density and current operators as

$$
\rho_{\mathbf{x}}=\sum_{\varepsilon= \pm} \rho_{\mathbf{x}, \varepsilon} \quad j_{\mathbf{x}}=\sum_{\varepsilon= \pm} \varepsilon \rho_{\mathbf{x}, \varepsilon}
$$

we get, as an immediate consequence of the commutation rules (0.2)

$\frac{\partial \widehat{\rho}_{t, p}}{d t}=e^{H t}\left[H, \widehat{\rho}_{p}\right] e^{-H t}=p v_{N}(p) \widehat{j}_{t, p} \quad \frac{\partial \widehat{j}_{t, p}}{d t}=e^{H t}\left[H, \widehat{j}_{p}\right] e^{-H t}=p v_{J}(p) \widehat{\rho}_{t, p}$

with

$$
v_{J}(p)=\left(1-\frac{\lambda \widehat{v}(p)}{2 \pi}\right) \quad v_{N}(p)=\left(1+\frac{\lambda \widehat{v}(p)}{2 \pi}\right)
$$


and we have used that, from the commutation rules $(0.2),\left[V, \rho_{p}\right]=\frac{\lambda \widehat{v}(p)}{2 \pi} \rho_{p}$, $\left[V, \rho_{p}\right]=-\frac{\lambda \widehat{v}(p)}{2 \pi} \rho_{p}$. As a consequence, the following Ward Identities [14] can be derived

$$
\begin{aligned}
& -i \omega\left\langle\widehat{\rho}_{\mathbf{p}} \widehat{\psi}_{\mathbf{k}, \varepsilon}^{-} \widehat{\psi}_{\mathbf{k}+\mathbf{p}, \varepsilon}^{+}\right\rangle_{T}+ \\
& \varepsilon v_{J}\left\langle\widehat{j}_{\mathbf{p}} \widehat{\psi}_{\mathbf{k}, \varepsilon}^{-} \widehat{\psi}_{\mathbf{k}+\mathbf{p}, \varepsilon}^{+}\right\rangle_{T}=\left\langle\widehat{\psi}_{\mathbf{k}, \varepsilon}^{-} \widehat{\psi}_{\mathbf{k}, \varepsilon}^{+}\right\rangle-\left\langle\widehat{\psi}_{\mathbf{k}+\mathbf{p}, \varepsilon}^{-} \widehat{\psi}_{\mathbf{k}+\mathbf{p}, \varepsilon}^{+}\right\rangle
\end{aligned}
$$

and

$$
\begin{aligned}
& -i \omega\left\langle\widehat{j}_{\mathbf{p}} \widehat{\psi}_{\mathbf{k}, \varepsilon}^{-} \widehat{\psi}_{\mathbf{k}+\mathbf{p}, \varepsilon}^{+}\right\rangle_{T}+ \\
& \varepsilon v_{N} p\left\langle\widehat{\rho}_{\mathbf{p}} \widehat{\psi}_{\mathbf{k}, \varepsilon}^{-} \widehat{\psi}_{\mathbf{k}+\mathbf{p}, \varepsilon}^{+}\right\rangle_{T}=\left\langle\widehat{\psi}_{\mathbf{k}, \varepsilon}^{-} \widehat{\psi}_{\mathbf{k}, \varepsilon}^{+}\right\rangle-\left\langle\widehat{\psi}_{\mathbf{k}+\mathbf{p}, \varepsilon}^{-} \widehat{\psi}_{\mathbf{k}+\mathbf{p}, \varepsilon}^{+}\right\rangle
\end{aligned}
$$

The quantities $v_{N}(p)$ and $v_{J}(p)$ are velocities associated with the charge and current excitations; they appear also related to the Drude weight $D$ and the susceptibility $\kappa[7]$ (see below) by the relations $D=\frac{v_{J}(0)}{\pi}$ and $\kappa=\frac{\pi}{v_{N}(0)}$ so that

$$
\frac{D}{\kappa}=v_{F}^{2}
$$

\subsection{Non solvable lattice models and the Luttinger liquid conjecture}

The exact solvability of the Luttinger model is due to certain peculiar features, like its linear "relativistic" dispersion relation, which are lost in more realistic models of one dimensional metals. It is then natural to ask which features of the Luttinger model are still true in more realistic lattice hamiltonian like the following tight-binding model

$$
\begin{aligned}
& H=H_{0}+V \\
& H_{0}=-\frac{1}{2} \sum_{x=1}^{L-1}\left[a_{x}^{+} a_{x+1}^{-}+a_{x+1}^{+} a_{x}^{-}\right]+\mu \sum_{x=1}^{L}\left(a_{x}^{+} a_{x}^{-}-\frac{1}{2}\right) \\
& V=\lambda \sum_{1 \leq x, y \leq L} v(x-y)\left(a_{x}^{+} a_{x}^{-}-\frac{1}{2}\right)\left(a_{y}^{+} a_{y}^{-}-\frac{1}{2}\right)
\end{aligned}
$$

where $a_{x}^{ \pm}, x=1,2, . ., L$, are fermionic creation or destruction operators, $\rho_{x}=a_{x}^{+} a_{x}^{-} \mu$ is the chemical potential, and $|v(x-y)| \leq e^{-\kappa|x-y|}$. Contrary to what happens in the Luttinger model, the hamiltonian of the chain model (0.34) cannot be rewritten as a quadratic boson Hamiltonian. An exact solution by Bethe ansatz [18] exists in the special case ( $X X Z$ model)

$$
v(x-y)=\delta_{|x-y|, 1} / 2 \quad \mu=0
$$


The solution provides the excitation spectrum and several thermodynamic properties but not an explicit form for the correlations.

We define $O_{\mathbf{x}, \varepsilon}=e^{H t} O_{x} e^{-H t}, \mathbf{x}=(t, x)$ and

$$
<O_{\mathbf{x}_{1} \ldots} \ldots O_{\mathbf{x}_{n}}>_{\beta}=\left.\frac{\operatorname{Tr} e^{-\beta H} \mathcal{T} O_{\mathbf{x}_{1} \ldots O_{\mathbf{x}_{n}}}}{\operatorname{Tr} e^{-\beta H}}\right|_{T}
$$

where $\mathcal{T}$ is the time ordering operator and $T$ denotes truncation (the label $T$ is understood in the l.h.s. of (0.36)); we call $\lim _{\beta \rightarrow \infty}<O_{\mathbf{x}_{1}} \ldots O_{\mathbf{x}_{n}}>_{\beta}=<$ $O_{\mathbf{x}_{1}} \ldots O_{\mathbf{x}_{n}}>$. In addition to the density $\rho_{x}$ it is convenient to introduce the current

$$
J_{x}=\frac{1}{2 i}\left[a_{x+1}^{+} a_{x}^{-}-a_{x}^{+} a_{x+1}^{-}\right] .
$$

From the continuity equation

$$
\frac{\partial \rho_{\mathbf{x}}}{\partial t}=e^{H t}\left[H, \rho_{x}\right] e^{-H t}=-i\left(J_{x, t}-J_{x-1, t}\right)
$$

where we have used that $\left[V, \rho_{x}\right]=0$, we can derive the following Ward Identity

$$
\begin{aligned}
& -i \omega<\widehat{\rho}_{\mathbf{p}} \widehat{a}_{\mathbf{k}}^{-} \widehat{a}_{\mathbf{k}+\mathbf{p}}^{+}>-i\left(1-e^{i p}\right)<J_{\mathbf{p}} \widehat{a}_{\mathbf{k}}^{-} \widehat{a}_{\mathbf{k}+\mathbf{p}}^{+}>= \\
& <\widehat{a}_{\mathbf{k}}^{-} \widehat{a}_{\mathbf{k}}^{+}>-<\widehat{a}_{\mathbf{k}+\mathbf{p}}^{-} \widehat{a}_{\mathbf{k}+\mathbf{p}}^{+}>
\end{aligned}
$$

The 1.h.s. of the above Ward Identity coincides with the one of the free $\lambda=0$ case, as consequence of $\left[V, \rho_{x}\right]=0$, while in its analogue for the Luttinger model (0.32) there is an explicit dependence from the coupling. The fact that the Luttinger model has an extra Ward Identity (0.33) is related to chiral gauge invariance which is absent in the model (0.34).

Other Ward Identities for the model (0.34) are

$$
\begin{aligned}
& -i \omega<\widehat{\rho}_{\mathbf{p}} \widehat{\rho}_{-\mathbf{p}}>-i\left(1-e^{-i p}\right)<\widehat{\rho}_{\mathbf{p}} \widehat{J}_{-\mathbf{p}}>=0 \\
& -i \omega<\widehat{\rho}_{\mathbf{p}} \widehat{J}_{-\mathbf{p}}>-i\left(1-e^{-i p}\right)<\widehat{J}_{\mathbf{p}} \widehat{J}_{-\mathbf{p}}>=i\left(1-e^{-i p}\right) \Delta .
\end{aligned}
$$

where $\Delta=<\Delta_{x}>$ and $\Delta_{x}=-\frac{1}{2}\left[a_{x}^{+} a_{x+1}^{-}+a_{x+1}^{+} a_{x}^{-}\right]$. Note the last term in the second of $(0.40)$, coming from the fact that $\left[\rho_{x}, J_{y}\right]=-i \delta_{x, y} \Delta_{x}+$ $i \delta_{x-1, y} \Delta_{y}$; it is called Schwinger term and is related to the diamagnetic part of the current $\Delta_{x}$, whose mean value $\left\langle\Delta_{x}\right\rangle$ is indeed independent of $x$.

The susceptibility is defined as, if $\mathbf{p}=(\omega, p)$

$$
\kappa=\lim _{p \rightarrow 0} \lim _{\omega \rightarrow 0}<\widehat{\rho}_{\mathbf{p}} \widehat{\rho}_{-\mathbf{p}}>
$$

while the conductivity is

$$
\sigma(\omega)=\frac{D(i \omega, p)}{i \omega+0^{+}} \quad D(\mathbf{p})=-\Delta-<\widehat{J}_{\mathbf{p}} \widehat{J}_{-\mathbf{p}}>
$$


while the Drude weight is $\lim _{\omega \rightarrow 0} D(\mathbf{p})=D$; a finite Drude weight implies an infinite dc conductivity.

The WI (0.40) and the fact that $<\rho_{\mathbf{p}} J_{-\mathbf{p}}>=<J_{\mathbf{p}} \rho_{-\mathbf{p}}>$ implies that

$$
<\rho_{\mathbf{p}} \rho_{-\mathbf{p}}>\left.\right|_{\omega, 0}=0 \quad, \quad D(0, p)=0 .
$$

The fact that $\kappa$ and $D$ are not vanishing is related to the fact that $<$ $\rho_{\mathbf{p}} \rho_{-\mathbf{p}}>$ and $D(\mathbf{p})$ are not continuous, a fact which can be easily checked in the non interacting case. Indeed if $\lambda=0$ the 2-point function $\left\langle a_{\mathbf{x}}^{-} a_{\mathbf{y}}^{+}>_{0} \equiv\right.$ $g(\mathbf{x}-\mathbf{y})$ is given by

$$
g(\mathbf{x}, \mathbf{y})=\int d \mathbf{k} \frac{e^{i \mathbf{k x}}}{-i k_{0}+\cos k-\cos p_{F}}=\int d \mathbf{k} e^{i \mathbf{k x}} \widehat{g}(\mathbf{k})
$$

with $\cos p_{F}=\mu ; \widehat{g}(\mathbf{k})$ is singular at $k_{0}=0$ and $k= \pm p_{F}$ and close to each one of the two Fermi points $\pm p_{F}$ the 2-point function is identical, up to small correction, to the Luttinger model non interacting two point function

$$
\widehat{g}\left(\mathbf{k}^{\prime} \pm \mathbf{p}_{F}\right)=\frac{1}{-i k_{0} \pm \sin p_{F} k^{\prime}}\left(1+O\left(\mathbf{k}^{\prime}\right)\right)
$$

for small $\mathbf{k}^{\prime}$, where $\mathbf{p}_{F}=\left(0, p_{F}\right)$. The density-density correlation in the non interacting case $\lambda=0$ is

$$
<\rho_{\mathbf{p}} \rho_{-\mathbf{p}}>_{0}=\int \frac{d k}{2 \pi} \frac{1}{-i p_{0}+\cos k-\cos (k+p)}\left[n_{F}(k)-n_{F}(k+p)\right]
$$

where $n_{F}(k)$ is the Fermi distribution; therefore for small $p$

$$
<\rho_{\mathbf{p}} \rho_{-\mathbf{p}}>_{0}=\frac{1}{\pi} \frac{\sin p_{F} p^{2}}{\omega^{2}+\sin ^{2} p_{F} p^{2}}\left(1+O\left(\frac{p^{3}}{|\mathbf{p}|^{2}}\right)\right.
$$

In the presence of interaction it is reasonable to expect, following [1], the presence of critical exponents but there is no reason to expect that they coincide with the Luttinger model ones. On the other hand, the exponents in the Luttinger model verify $(0.22),(0.24),(0.27)$ and the content of the Luttinger liquid conjecture [8], [7],[6] is that the same relations are true in a wide class of models, including models with non linear bands like the model (0.34). In particular according to such conjecture there exists a (model dependent) function $K$ such that

$$
\eta=\frac{1}{2}\left[K+K^{-1}-2\right] \quad X_{+}=K \quad X_{-}=K^{-1}
$$

and $K$ can be obtained from thermodynamic functions by the relations

$$
\kappa=\frac{K}{\pi v_{F}} \quad D=\frac{v_{F} K}{\pi}
$$


where $v_{F}$ is the interacting Fermi velocity.

As the validity of (0.48), (0.49) in the Luttinger model relies on its exact mapping in a system of free bosons (a property which is true only for its linear dispersion relation), their validity in the model (0.2), with a sinusoidal dispersion relation, is far from to be obvious. A very important test came from the exact solution of the $X X Z$; indeed by the Bethe ansatz solution it is found [18], [7],[46], if $\cos \bar{\mu}=\lambda$

$$
v_{F}=\frac{\pi}{\bar{\mu}} \sin \bar{\mu} \quad \kappa=\frac{1}{2 \pi(\pi / \bar{\mu}-1) \sin \bar{\mu}}
$$

and

$$
D=\frac{\pi}{\bar{\mu}} \frac{\sin \bar{\mu}}{2(1-\bar{\mu} / \pi))}
$$

Even if such expressions are different (as functions of the interaction) from the Luttinger ones, the relation (0.33) follows. Moreover in [17] the correlation length exponent $\nu$ in the XYZ model has been computed finding $\nu=\frac{\pi}{2 \bar{\mu}}$; therefore using the scaling relation $\nu=\left(2-X_{+}^{-1}\right)$ proposed in [8] we can computed the other exponents, for instance

$$
X_{+}=K=\left(2-\frac{2 \bar{\mu}}{\pi}\right)^{-1}=1+\frac{2 \lambda}{\pi}+O\left(\lambda^{2}\right)
$$

The same values is found by the value of $v_{F}$ and $D$ using (0.49), and this provides a check of the Luttinger liquid conjecture, as stressed in [7].

In the model (0.34) with a generic short range interaction there is no exact solution. To give support to the validity of the Luttinger liquid relations (0.48), (0.49) in a wide class of models, including non solvable ones, in [6] a perturbation of the Luttinger model was considered in which the linear dispersion relation is replaced by

$$
\varepsilon_{ \pm}(k)= \pm k+\frac{1}{2 m} k^{2}+\frac{\lambda}{12 m^{2}} k^{3}
$$

with $\lambda \geq \frac{3}{4}$. While such model reduces to the Luttinger model as $m \rightarrow \infty$, for finite $m$ non quadratic terms appear in the boson hamiltonian; however remarkably the Luttinger liquid relations (0.48), (0.49) still holds. This provides evidence in support of the conjecture, but on the other hand the Luttinger liquid relations could be checked up to $O\left(\mathrm{~m}^{-2}\right)$ and the model with dispersion relation (0.53) is still only an approximate effective description for the model (0.34).

Instead of using boson variables, one could try to prove the Luttinger liquid relations (0.48), (0.49) using an expansion in Feynman graphs, see 
e.g. [20]; its use is however problematic for 1D interacting fermions as the expansion is plagued by infrared divergences. Dzylaloshinski and Larkin [21] considered 1D fermions with bandwidth cut-off; contrary to the Luttinger model this model, called Tomonaga model, is not solvable. They performed a resummations of the perturbative expansions finding the anomalous decay of correlations, but at the price of several (uncontrolled) approximations. The use of (multiplicative) Renormalization Group methods for the Tomonaga model dates back to Solyom [22]; irrelevant terms are simply discarded and the presence of anomalous exponents results from a property, called vanishing of beta function, which was proved only at lowest orders in perturbation theory. Metzner and Di Castro [14] pointed out that the vanishing of the Beta function in the multiplicative RG follows from the Ward Identities (0.32), (0.33), which are however true only in the Luttinger and not in the Tomonaga model.

With respect to multiplicative RG, exact Wilsonian [23] RG method, used by Polchinski [24] and Gallavotti [25] for the renormalization of $\phi^{4}$ models and therefore applied by to non relativistic interacting fermions in $d=1,3$ by Benfatto and Gallavotti [26], has two main advantages. They are : (i) exact, in the sense that it does not need any relativistic approximation or continuum limit and it allow us to keep the full lattice structure of the problem; (ii) non-perturbative, in the sense that it involves expansions whose convergence can be mathematically proved. The fact that such methods are exact and can take into account the irrelevant terms is a crucial feature: the Luttinger or the lattice model (0.34) differ for irrelevant terms and the proof of the Luttinger liquid relations (0.48), (0.49) consists essentially in showing that such irrelevant terms, while contributing to the exponents or other physical quantities, leave unchanged the Luttinger liquid relations.

Such exact RG methods have been used in Constructive Quantum Field Theory to prove the existence of the continuum limit of Quantum Theory model at $d=1+1$ dimensions, like the massless Gross-Neveu model with $N>1$ colors [27], [28] or the massive Yukawa model [29]. The main extra difficulty in analyzing a model like (0.2), with respect to QFT model like the Gross-Neveu model analyzed in [27], is that the theory is not asymptotically free; it belongs to a class of models with vanishing beta function. Such models can be constructed only exploiting non trivial cancellations at all orders in the renormalized expansion; this is a crucial difference with respect to the asymptotically free models in which a second order 
computation is enough for establishing the nature of the flow of the effective coupling. The first example of rigorous construction of a model with vanishing beta function was in [30] and it regards the Jellium model in $1 \mathrm{D}$, describing interacting non relativistic fermions in the continuum. The crucial property of vanishing of Beta function was proved using an indirect argument based on comparison with the exact solution of the Luttinger model [12]. The construction of models with vanishing Beta function without any use of exact solutions was finally realized in $[31 ; 32$; 33] solving the well known technical problem related to the basic conflict between Wilsonian Renormalization Group and Ward Identities; the decomposition in momentum space necessary in Wilson scheme breaks the local gauge invariance necessary to establish Ward Identities.

In the following sections we will resume the main achievements of the application of such exact Renormalization Group methods to systems like (0.34) and the main steps of the proof of the Luttinger liquid relations $(0.48),(0.49)$ for non solvable models, finally achieved in $[34 ; 35 ; 36 ; 37$; $38]$.

\subsection{Exact Renormalization Group analysis}

The starting point of the exact Renormalization Group analysis is the expression of the correlations of the lattice model (0.34) in terms of Grassmann integrals. We introduce a set of Grassmann variables $\psi_{\mathbf{x}}^{ \pm}$such that $\left\{\psi^{ \pm}, \psi^{ \pm}\right\}=\left\{\psi^{ \pm}, \psi^{\mp}\right\}=0$ and

$$
\int d \psi_{\mathbf{x}}^{ \pm}=0 \quad \int d \psi_{\mathbf{x}}^{ \pm} \psi_{\mathbf{x}}^{ \pm}=0
$$

The integral of any analytic functions can be obtained by (0.54) by linearity. The correlations of a model like (0.34) can be written by suitable derivatives of generating functional expressed by the following Grassman integral

$$
e^{\mathcal{W}(A, \phi)}=\int P(d \psi) e^{-\mathcal{V}(\psi)+B(A, \psi)+\int d \mathbf{x}\left[\phi_{\mathbf{x}}^{+} \psi_{\mathbf{x}}^{-}+\psi_{\mathbf{x}}^{-} \psi_{\mathbf{x}}^{+}\right]}
$$

where $\phi_{\mathbf{x}}^{ \pm}$are Grassmann variables, $\int d \mathbf{x}$ is a shortcut for $\sum_{x} \int_{-\beta / 2}^{\beta / 2} d x_{0}$, $P(d \psi)$ is a Grassmann Gaussian measure in the field variables $\psi_{\mathbf{x}}^{ \pm}$with covariance (the free propagator) given by

$$
g(\mathbf{x}-\mathbf{y})=\frac{1}{\beta L} \sum_{\mathbf{k}} \frac{e^{i \mathbf{k}(\mathbf{x}-\mathbf{y})}}{-i k_{0}+\left(\cos p_{F}-\cos k\right)},
$$


with

$$
\cos p_{F}=-\lambda-\mu-\nu
$$

and the interaction is

$$
\mathcal{V}(\psi)=\lambda \int d \mathbf{x} d \mathbf{y} v(\mathbf{x}-\mathbf{y}) \psi_{\mathbf{x}}^{+} \psi_{\mathbf{y}}^{+} \psi_{\mathbf{y}}^{-} \psi_{\mathbf{x}}^{-}+\nu \int d \mathbf{x} \psi_{\mathbf{x}}^{+} \psi_{\mathbf{x}}^{-}
$$

with $\mathbf{e}_{\mathbf{1}}=(0,1), v(\mathbf{x}-\mathbf{y})=\delta(t-s) v(x-y)$ while the source is

$$
B(A, \psi)=\int d \mathbf{x}\left\{\psi_{\mathbf{x}}^{+} \psi_{\mathbf{x}}^{-} A_{0}(\mathbf{x})+\frac{1}{2 i}\left[\psi_{\mathbf{x}+\mathbf{e}_{1}}^{+} \psi_{\mathbf{x}}^{-}-\psi_{\mathbf{x}}^{+} \psi_{\mathbf{x}+\mathbf{e}_{1}}^{-}\right] A_{1}(\mathbf{x})\right\}
$$

Contrary to what happens in the Luttinger model, the value of the Fermi momentum is modified by the interaction; it is convenient to analyze (0.55) considering $p_{F}$ as a parameter, choosing $\nu$ as a function of $\lambda$ and $p_{F}$ so that the singularity of the Fourier transform of the interacting two-point function is fixed at $\mathbf{k}=\left( \pm p_{F}, 0\right)$. Finally from (0.57) we get the Fermi momentum $p_{F}$ as function of $\lambda$ and $\mu$.

We briefly describe the exact Renormalization Group analysis of (0.55), as developed in [31]; for a tutorial introduction to the properties of Grassman integrals, we refer to [39]. The starting point is to use the addition property for a gaussian Grassmann measure saying that

$$
\int P(d \psi) F(\psi)=\int P\left(d \psi^{(1)}\right) \int P\left(d \psi^{(2)}\right) F\left(\psi^{(1)}+\psi^{(2)}\right)
$$

where $P(d \psi), P\left(d \psi^{(1)}\right), P\left(d \psi^{(2)}\right)$ are Grassmann gaussian measures respectively with propagator $g, g^{(1)}, g^{(2)}$ and $g=g^{(1)}+g^{(2)}$. We can introduce the following smooth decomposition of the unity

$$
1=\sum_{\varepsilon= \pm} \chi\left(k-\varepsilon p_{F}, k_{0}\right)+f_{1}(\mathbf{k})
$$

where $\chi\left(k^{\prime}, k_{0}\right)$ is a smooth compact-support function such that $\chi\left(k^{\prime}, k_{0}\right)=$ 1 for $\left|\mathbf{k}^{\prime}\right|_{T^{1}} \leq t_{0}$ and $\chi\left(k^{\prime}, k_{0}\right)=0$ for $\left|\mathbf{k}^{\prime}\right|_{T^{1}} \geq \gamma t_{0}$, if $t_{0}, \gamma>1$ are suitable constants; in other words, $\chi\left(k-\varepsilon p_{F}, k_{0}\right)$ is a compact support function non-vanishing around the two Fermi points $\left(0, \pm p_{F}\right)$. We can decompose the propagator as

$$
\widehat{g}(\mathbf{k})=\sum_{\varepsilon= \pm} \chi\left(k-\varepsilon p_{F}, k_{0}\right) \widehat{g}(\mathbf{k})+f^{(1)}(\mathbf{k}) \widehat{g}(\mathbf{k}) \equiv \sum_{\varepsilon= \pm} \widehat{g}_{\varepsilon}^{(\leq 0)}(\mathbf{k})+\widehat{g}^{(1)}(\mathbf{k})
$$

leading, according to (0.60), to a decomposition of the Grassmann field of the form

$$
\psi_{\mathbf{x}}=\sum_{\varepsilon= \pm} e^{i \varepsilon p_{F} x} \psi_{\mathbf{x}, \varepsilon}^{(\leq 0)}+\psi_{\mathbf{x}}^{(1)}
$$


where $\psi_{\mathbf{x}, \varepsilon}^{(\leq 0)}, \psi_{\mathbf{x}}^{(1)}$ are independent Grassmann fields with propagator $e^{\left.i \varepsilon p_{F}(x-y)\right)} \widehat{g}_{\varepsilon}^{(\leq 0)}(\mathbf{x}-\mathbf{y})$ and $\widehat{g}^{(1)}(\mathbf{x}-\mathbf{y})$ respectively. Note that, while the propagator of the fields $\psi_{\mathbf{x}, \varepsilon}^{(\leq 0)}$ has a slow $O\left(|\mathbf{x}-\mathbf{y}|^{-1}\right)$ decay for large distances, the propagator of $\psi_{\mathbf{x}}^{(1)}$ decays faster than any power; that is for any $N$

$$
\left|g^{(1)}(\mathbf{x}-\mathbf{y})\right| \leq \frac{C_{N}}{1+|\mathbf{x}-\mathbf{y}|^{N}}
$$

Using (0.60) we can rewrite (0.55) in the following way (in the $\phi=0$ case for simplicity)

$$
e^{\mathcal{W}(A, 0)}=\int P\left(d \psi^{\leq 0}\right) \int P\left(d \psi^{(1)}\right) e^{-\mathcal{V}\left(\psi^{(0)}+\psi^{(1)}\right)+B\left(A, \psi^{(\leq 0)}+\psi^{(1)}\right)}
$$

where $\quad P\left(d \psi^{\leq 0}\right) \quad$ and $\quad P\left(d \psi^{(1)}\right) \quad$ have respectively propagator $\sum_{\varepsilon= \pm} e^{i \varepsilon p_{F}(x-y)} g_{\varepsilon}^{(\leq 0)}(\mathbf{x}-\mathbf{y})$ and $g^{(1}(\mathbf{x}-\mathbf{y})$. The integration of the "high energy" degrees of freedom can be done using the invariance of exponential property

$$
\int P(d \psi) e^{X(\psi+\phi)}=\exp \left[\sum_{n=0}^{\infty} \frac{1}{n !} \mathcal{E}^{T}(X(.+\phi ; n))\right]=e^{X^{\prime}(\phi)}
$$

where $\mathcal{E}^{T}(X(.+\phi ; n))$ are the fermionic truncated expectation, which are given by the sum over all the possible connected Feynman graphs (see Fig. 0.1). By using (0.66) we get

$$
e^{\mathcal{W}(A, 0)}=\int P\left(d \psi^{\leq 0}\right) e^{-\mathcal{V}^{(0)}\left(\psi^{(0)}\right)+B^{(0)}\left(A, \psi^{(\leq 0)}\right)}
$$

where $\mathcal{V}^{(0)}\left(\psi^{(\leq 0)}\right)$ and $B^{(0)}\left(A, \psi^{(\leq 0)}\right)$ is sum of monomials of any degree multiplied by suitable kernels; in particular $\mathcal{V}^{(0)}\left(\psi^{(\leq 0)}\right)$ is given by

$$
\mathcal{V}^{(0)}\left(\psi^{(0)}\right)=\sum_{n=2}^{\infty} W_{n}^{(0)}\left(\mathbf{x}_{1}, \ldots, \mathbf{x}_{n}\right) \prod_{i=1}^{n} \psi_{\mathbf{x}_{i}, \varepsilon_{i}}^{\sigma_{i}}
$$

and a similar expression holds for $B^{(0)}\left(A, \psi^{(\leq 0)}\right)$ with kernels $W_{n, m}^{(0)}$ with $n$ $\psi$ and $m A$ fields.

The kernels $W_{n, m}^{(0)}$ are analytic functions of $\lambda$ and $\nu$ in a complex disk, and we describe briefly how such a non-perturbative property is obtained. According to (0.66), the kernels $W_{n}^{(0)}$ can be written as power series in $\lambda, \nu$, and each order $k$ can be written as sum of connected Feynman graphs with $\lambda$ and $\nu$ vertices and propagators $g^{(1)}(\mathbf{x})$; by $(0.64)$, it is easy to check that each Feynman graph at order $k$ is bounded by $k !^{-1}[\max (\lambda, \nu)]^{k} C^{k}$, for a suitable constant $C$. This is however not enough for proving the 


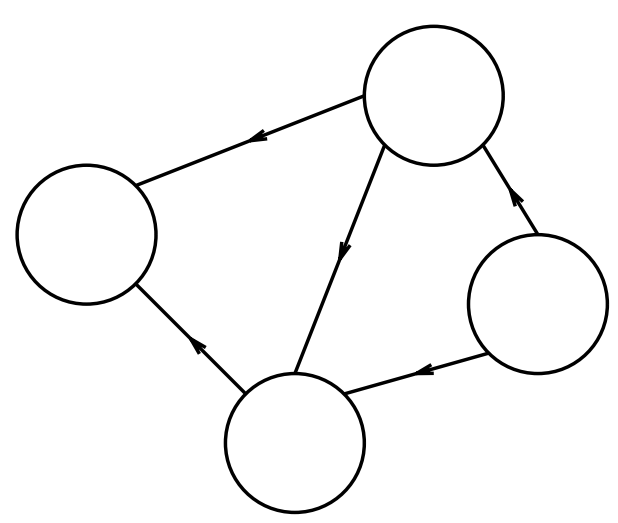

Fig. 0.1 An example of connected Feynman graph contributing to $\mathcal{E}^{T}$

convergence of the expansion for $W_{n}^{(0)}$, as the number of graphs contributing to order $k$ are $O\left(k !^{2}\right)$ so that the $k$-order contribution is bounded by $k ![\max (\lambda, \nu)]^{k} C^{k}$. The presence of such $k !$ apparently prevent the convergence of the series. While in the case of bosonic models such factorial is really there, in the case of fermionic systems cancellations between Feynman diagrams (ultimately related to the fermionic anticommutativity properties) have the effect that the real final bound is much better and convergence of the series for $W_{n}^{(0)}$ can be finally achieved [40]. Indeed the fermionic expectation can be written as a determinant

$$
\int P(d \psi) \psi_{\mathbf{x}_{1}}^{-(1)} \ldots \psi_{\mathbf{x}_{n}}^{-(1)} \psi_{\mathbf{y}_{1}}^{+(1)} \ldots \psi_{\mathbf{y}_{n}}^{+(1)}=\operatorname{Det} G
$$

where $G_{i, j}=g^{(1)}\left(\mathbf{x}_{i}-\mathbf{y}_{i}\right)$; writing $g^{(1)}\left(\mathbf{x}_{i}-\mathbf{y}_{i}\right)$ in the form

$$
g^{(1)}(\mathbf{x}-\mathbf{y})=\int d \mathbf{z} A^{(1)}(\mathbf{x}-\mathbf{z}) \bar{B}^{(1)}(\mathbf{y}-\mathbf{z})=(A, B)
$$

then, if $(A, A)$ and $(B, B) \leq C$ by the Gram inequality

$$
|\operatorname{Det} G| \leq C^{n}
$$

Note that, even if $\operatorname{Det} G$ is expressed by $n$ ! terms, in the final bound no $n$ ! appear. This fact is however still not enough; in order to integrate over 
the coordinates one needs to exploit the decay properties of the propagator (0.64), which are lost in the bound (0.71). One needs to extract from the truncated expectation a minimal set of propagators ensuring the connection between vertices (which allow the integration over coordinates) leaving all the other fields grouped in determinants, so that they can be bounded by the Gram bound. This is made possible by the Battle-Brydges-Federbush formula [41] (see for instance again [39] for a derivation); the truncated expectation is written not as sum of Feynman diagrams, but sum of trees of propagators times determinants (represented in the Fig.0.2 as uncoupled lines). The trees of propagators are used to perform the integration over the coordinates and the determinants are bounded by Gram bounds, so that the convergence of the expansion for the kernels $W_{n}^{(0)}$ for $\lambda, \nu$ not too large follows.

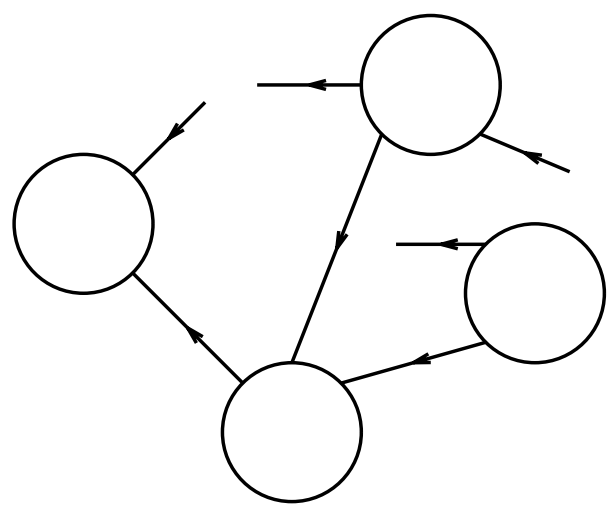

Fig. 0.2 One of the term in the Battle-Brydges-Federbush formula

The integration of the high energy fields $\psi^{(1)}$ says that the generating function on the model (0.34), namely (0.55), can be exactly rewritten as (0.67), that is in terms of two kinds of fermions with a band-width cut-off around the two Fermi points. The Tomonaga approximation corresponds to neglecting in $\mathcal{V}^{(0)}$ the irrelevant terms with $n \geq 4$ in $\mathcal{V}^{(0)}$ and in replacing $\cos \left(k^{\prime}+ \pm p_{F}\right)-\cos p_{F}$ with $\pm\left(\sin p_{F}\right) k^{\prime}$; the exact $\mathrm{RG}$ analysis below allows to take such terms into account. It is natural to continue the analysis of (0.67) again integrating the momenta closer and closer to the Fermi points. 
We can write

$$
\chi\left(k-\varepsilon p_{F}, k_{0}\right)=\sum_{j=-\infty}^{0} f_{j}\left(\mathbf{k}^{\prime}\right)
$$

where $\mathbf{k}^{\prime}=\mathbf{k}-\varepsilon \mathbf{p}_{F}, f_{j}\left(\mathbf{k}^{\prime}\right)$ (see Fig.0.3) is a compact support function with support in $t_{0} \gamma^{j-1} \leq\left|\mathbf{k}^{\prime}\right|_{T^{1}} \leq t_{0} \gamma^{j+1}, \mathbf{p}_{F}=\left(0, p_{F}\right)$ and $t_{0}, \gamma>1$ are suitable constants. We can write the propagator $\widehat{g}_{\varepsilon}^{(\leq 0)}$ as

$$
\widehat{g}_{\varepsilon}^{(\leq 0)}\left(\mathbf{k}^{\prime}\right)=\sum_{j=-\infty}^{0} \widehat{g}_{\varepsilon}^{(j)}\left(\mathbf{k}^{\prime}\right)
$$

where $\widehat{g}_{\varepsilon}^{(\leq 0)}\left(\mathbf{k}^{\prime}\right)$ is a propagator living at momentum scales $O\left(\gamma^{j}\right)$ close one of the two Fermi points. The above decomposition of the propagator induced an analogous decomposition of the fermionic fields as sum of fields $\psi^{(\leq 0)}=\sum_{h=-\infty}^{0} \psi^{(h)}$.

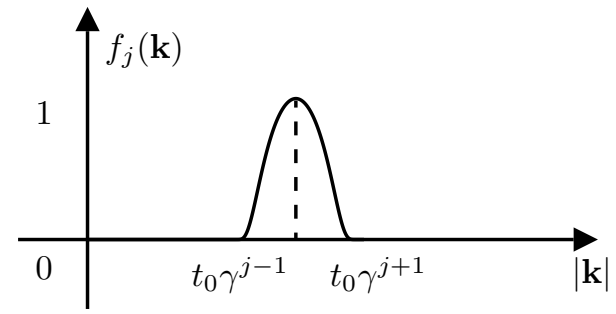

Fig. 0.3 The function $f_{j}(\mathbf{k})$

After the integration of the fields $\psi_{\varepsilon}^{(0)}, \psi_{\varepsilon}^{(-1)}, . ., \psi_{\varepsilon}^{(h+1)}$ by the reiterate application of (0.66) we get

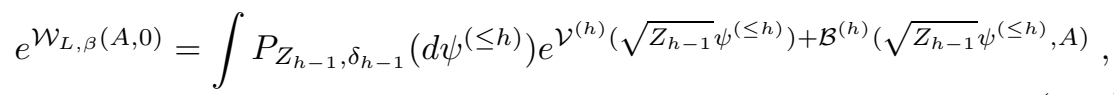

where $P_{Z_{h}, \delta_{h}}\left(d \psi^{(\leq h)}\right)$ is the Gaussian Grassmann integration with propagator

$$
\widehat{g}_{\varepsilon}^{(\leq h)}\left(\mathbf{k}^{\prime}\right)=\frac{\chi_{h}\left(\mathbf{k}^{\prime}\right)}{Z_{h}} \frac{1}{-i k_{0}+\left(1+\delta_{h}\right)\left(\cos p_{F}-\cos \left(k^{\prime}+\varepsilon p_{F}\right)\right)}
$$

with $\chi_{h}\left(\mathbf{k}^{\prime}\right)$ a smooth compact support function non vanishing for $\left|\mathbf{k}^{\prime}\right| \leq$ $t_{0} \gamma^{h}, Z_{h}$ is the wave function renormalization and $v_{h}=\sin p_{F}\left(1+\delta_{h}\right)$ is the effective Fermi velocity; finally the effective interaction $\mathcal{V}^{(h)}(\psi)$ is a sum over monomials in the Grassmann variables

$$
\mathcal{V}^{(h)}(\psi)=\gamma^{h} \nu_{h} F_{\nu}^{(h)}+\lambda_{h} F_{\lambda}^{(h)}+R_{h},
$$


where

$$
\begin{aligned}
& F_{\nu}^{(h)}=\sum_{\varepsilon= \pm} \int d \mathbf{x} \psi_{\mathbf{x}, \varepsilon}^{(\leq h)+} \psi_{\mathbf{x}, \varepsilon}^{(\leq h)-} \\
& F_{\lambda}^{(\leq h)}=\int d \mathbf{x} \psi_{\mathbf{x},+}^{(\leq h)+} \psi_{\mathbf{x},-}^{(\leq h)+} \psi_{\mathbf{x},+}^{(\leq h)-} \psi_{\mathbf{x},-}^{(\leq h)-}
\end{aligned}
$$

and $R^{(h)}$ is sum over monomials with $n \psi$ fields with negative scaling dimension $D$, defined by

$$
D=2-\frac{n}{2}
$$

In the same way (for $A^{(1)}=0$ for definiteness)

$$
\begin{aligned}
& \mathcal{B}^{(h)}\left(\sqrt{Z_{h-1}} \psi^{(\leq h)}, A\right)= \\
& \sum_{\varepsilon}\left[\int d \mathbf{x} Z_{h-1}^{(1)} A_{\mathbf{x}}^{(0)} \psi_{\mathbf{x}, \varepsilon}^{+(\leq h)} \psi_{\mathbf{x}, \varepsilon}^{-(\leq h)}+Z_{h-1}^{+} A_{\mathbf{x}}^{(0)} e^{2 i \varepsilon p_{F} x} \psi_{\mathbf{x}, \varepsilon}^{+(\leq h)} \psi_{\mathbf{x},-\varepsilon}^{-(\leq h)}\right]+\bar{R}_{h},
\end{aligned}
$$

and $\bar{R}_{h}$ is sum over monomials with $\mathrm{n} \psi$ fields and $m A$ fields with negative scaling dimension $D=2-\frac{n}{2}-m$. A similar expression holds setting $A^{(0)}=0$, and the corresponding renormalization constants will be called $Z_{h-1}^{(2)}, \widehat{Z}_{h-1}^{(+)}$.

It is natural the interpretation of $\lambda_{h}$ in (0.77) as the effective coupling of the model and of $\nu_{h}$ is the renormalization of the chemical potetntial. In the Renormalization Group language, the terms with positive or vanishing dimension are called relevant or marginal terms, respectively; the terms in $R_{h}$ or $\bar{R}_{h}$, having negative scaling dimension, are irrelevant. The kernels $W_{n, m}^{(h)}$ are expressed by expansions in the effective couplings $\lambda_{j}, \nu_{j}, j=$ $1,0,-1 \ldots, h$ which are convergent, by the Gram bound, provided that $\lambda_{j}, \nu_{j}$ are not too large; from them an expansion for the correlations and the other physical observables in terms of $\lambda_{j}, \nu_{j}$ is obtained. Such renormalized expansions in the effective couplings $\lambda_{j}, \nu_{j}$ should not be confused with the naive perturbative expansion of the correlations in $\lambda, \nu$; while the latter is plagued by infrared divergences the renormalized one is finite and even convergent provided that $\lambda_{j}, \nu_{j}$ are not too large uniformly in $j$. One has then to analyze the flow of the effective parameters.

Note first that $\nu_{j}$ is a relevant coupling and it is possible to choose the parameter $\nu$, by a fixed point argument, so that $\nu_{j}=O\left(\gamma^{j}\right)$; one can also prove, due to symmetry cancellations in the flow equation, that $\delta_{j} \rightarrow j \rightarrow-\infty \delta_{-\infty}=O(\lambda)$. In order to study the flow of $\lambda_{j}$ one first notices that the single scale propagator can be decomposed, for $h \leq 0$, as

$$
g_{\rho}^{(h)}(\mathbf{x}, \mathbf{y})=g_{T, \varepsilon}^{(h)}(\mathbf{x}, \mathbf{y})+r_{\varepsilon}^{(h)}(\mathbf{x}, \mathbf{y})
$$


where

$$
g_{T, \varepsilon}^{(h)}(\mathbf{x}, \mathbf{y})=\frac{1}{L^{2}} \sum_{\mathbf{k}} e^{-i \mathbf{k}(\mathbf{x}-\mathbf{y})} \frac{1}{Z_{h}} \frac{1}{-i k_{0}+\varepsilon v_{F} k},
$$

with $v_{F}=\sin p_{F}\left(1+\delta_{-\infty}\right)$ and for any $N$

$$
\left|r_{\varepsilon}^{(h)}(\mathbf{x}-\mathbf{y})\right| \leq C_{N} \frac{\gamma^{2 h}}{1+\left(\gamma^{h}|\mathbf{x}-\mathbf{y}|^{N}\right)}
$$

Note that $g_{T, \varepsilon}^{(h)}(\mathbf{x}, \mathbf{y})$ verifies bound similar to $(0.82)$ with $\gamma^{2 h}$ in the numerator replaced by $\gamma^{h}$; this means that we can rewrite the propagator as a Dirac propagator at scale $h$ plus a correction $r^{(h)}$ whose size is $O\left(\gamma^{2 h}\right)$ instead of $O\left(\gamma^{h}\right)$, that is much smaller. The above decomposition induces a similar decomposition in the flow equation; we can write

$$
\lambda_{j-1}=\lambda_{j}+\bar{\beta}_{\lambda}^{(j)}\left(\lambda_{j} ; \ldots ; \lambda\right)+O\left(\lambda^{2} \gamma^{j}\right)
$$

where in the last term we have included all the irrelevant contributions, containing at least an $r_{\varepsilon}^{(k)}$ or a $\nu_{k}$ or a $\mathcal{R} \mathcal{V}^{(0)}$, and it is asymptotically vanishing for dimensional reasons. The flow of $\lambda_{j}$ is then driven by $\bar{\beta}_{\lambda}^{(j)}$ with is expressed by a convergent series with each term sum of $O(1)$ renormalized Feynman graphs. A lowest order computation shows that such graphs cancel out; this is true to all orders (but of course it cannot be checked by an explicit computation) as a consequence of the following non-perturbative property, called vanishing of the beta function

$$
\left|\bar{\beta}_{\lambda}^{(j)}\left(\lambda_{j}, \ldots, \lambda_{j}\right)\right| \leq C \lambda_{j}^{2} \gamma^{j}
$$

We will briefly resume the main steps of the proof of $(0.84)$, which is the key of all the Renormalization Group analysis, in the following section. Assuming (0.84), it follows that the sequence $\lambda_{j}$ converges, as $j \rightarrow-\infty$, to an analytic function

$$
\lambda_{-\infty}(\lambda)=\lambda\left[\widehat{v}(0)-\widehat{v}\left(2 p_{F}\right)\right]+O\left(\lambda^{2}\right)
$$

such that $\left|\lambda_{j}-\lambda_{-\infty}\right| \leq C \lambda^{2} \gamma^{j}$. The effective wave function renormalization $Z_{j}$ verifies the following equation

$$
\frac{Z_{j-1}}{Z_{j}}=1+\bar{\beta}_{z}^{(j)}\left(\lambda_{j}, \ldots, \lambda_{0}\right)+O\left(\lambda \gamma^{j}\right)
$$

where again the last term in the r.h.s. includes the irrelevant terms and

$$
Z_{j} \sim A_{j}(\lambda) \gamma^{\eta j} \quad \eta=\log _{\gamma}\left(1+\bar{\beta}_{z}^{(-\infty)}\left(\lambda_{-\infty}, \ldots, \lambda_{-\infty}\right)\right.
$$


and $A_{j} \rightarrow_{j \rightarrow-\infty} A$ as $O\left(\lambda \gamma^{j}\right)$. Note that the exponent $\eta$ is written as power series in the variable $\lambda_{-\infty} / v_{F}$ and it depends only from $\bar{\beta}_{z}^{(j)}$ and not from the irrelevant term; on the contrary $A(\lambda)$ depends also on the irrelevant part of the flow equation. Similarly, $Z_{j}^{(i)}, i=1,2$ appearing in (0.79)

$$
\frac{Z_{j-1}^{(i)}}{Z_{j}}=1+\bar{\beta}_{1}^{(j)}\left(\lambda_{j}, \ldots, \lambda_{0}\right)+O\left(\lambda \gamma^{j}\right)
$$

and $\beta_{1}^{(j)}$ is asymptotically vanishing

$$
\left|\bar{\beta}_{ \pm}^{(j)}\left(\lambda_{j}, \ldots, \lambda_{j}\right)\right| \leq C \lambda^{2} \gamma^{j}
$$

with

$$
Z_{j}^{(1)} \sim A_{1, j} 2^{\eta j} \quad Z_{j}^{(2)} \sim A_{2, j} 2^{\eta j}
$$

Note that, while the exponents of $Z_{j}^{(1)}$ and $Z_{j}^{(2)}$ are the same (and equal to $\eta$ ), the amplitudes $A_{1}$ and $A_{2}$ are different. Finally $Z_{j}^{(2)} \sim \gamma^{\eta_{2} j}$ with again $\eta_{2}$ is written as power series in the variable $\lambda_{-\infty} / v_{F}$.

By taking derivatives with respect to the external fields $\phi^{ \pm}$we get that the 2-point function can be written as

$$
\left\langle a_{\mathbf{x}}^{-} a_{\mathbf{0}}^{+}\right\rangle=\left[\sum_{h=-\infty}^{0} \sum_{\varepsilon= \pm} e^{i \varepsilon p_{F}(x-y)} g_{\varepsilon}^{(h)}(\mathbf{x}-\mathbf{y})+g^{(1)}(\mathbf{x}-\mathbf{y})\right](1+O(\lambda))
$$

The above expression says that the 2-point function is sum of single scale propagators, each one with its wave function renormalization $Z_{h}$ and an effective fermi velocity $v_{h}=\sin p_{F}\left(1+\delta_{h}\right)$. From the above expression one can derive the large distance decay

$$
\left\langle a_{\mathbf{x}}^{-} a_{\mathbf{0}}^{+}\right\rangle_{T} \sim g_{0}(\mathbf{x}) \frac{1+\lambda f(\lambda)}{\left(x_{0}^{2}+v_{F}^{2} x^{2}\right)^{(\eta / 2)}},
$$

where $f(\lambda)$ is a bounded function, $\eta=b \lambda^{2}+O\left(\lambda^{3}\right)$, with $b>0$, and

$$
\begin{gathered}
g_{0}(\mathbf{x})=\sum_{\varepsilon= \pm} \frac{e^{i \varepsilon p_{F} x}}{-i x_{0}+\varepsilon v_{F} x} \\
v_{F}=\sin p_{F}+O(\lambda) \quad p_{F}=\cos ^{-1}(\mu+\lambda)+O(\lambda) .
\end{gathered}
$$

In momentum space, the meaning of (0.91) is even more transparent; it simply says, due to the compact support properties of the propagator, that for momenta close to the Ferm points $\mathbf{k}^{\prime} \sim t_{0} \gamma^{j}, \mathbf{k}=\mathbf{k}^{\prime}+\varepsilon \mathbf{p}_{F}, j \leq 0$ the 2-point function is essentially given by $\frac{\left|\mathbf{k}^{\prime}\right|^{\eta}}{-i k_{0}+\varepsilon v_{F} k^{\prime}}$, while for momenta far from the Fermi point the 2-point function is essentially given by $\widehat{g}^{(1)}(\mathbf{k})$, 
that is it has the same behavior of the non interacting one. A similar expansion holds for the density-density correlation which is given by

$$
\begin{gathered}
\left\langle\rho_{\mathbf{x}} \rho_{\mathbf{0}}\right\rangle_{T} \sim \cos \left(2 p_{F} x\right) \Omega^{3, a}(\mathbf{x})+\Omega^{3, b}(\mathbf{x}), \\
\Omega^{3, a}(\mathbf{x})=\frac{1+A_{1}(\mathbf{x})}{2 \pi^{2}\left[x^{2}+\left(v_{F} x_{0}\right)^{2}\right]_{+}}, \\
\Omega^{3, b}(\mathbf{x})=\frac{1}{2 \pi^{2}\left[x^{2}+\left(v_{F} x_{0}\right)^{2}\right]}\left\{\frac{x_{0}^{2}-\left(x / v_{F}\right)^{2}}{x^{2}+\left(v_{F} x_{0}\right)^{2}}+A_{2}(\mathbf{x})\right\},
\end{gathered}
$$

with $\left|A_{1}(\mathbf{x})\right|,\left|A_{2}(\mathbf{x})\right| \leq C|\lambda|$ and $X_{+}=1-a_{1} \lambda+O\left(\lambda^{2}\right)$ with

$$
X_{+}=1-\eta_{+}+\eta=1-a_{1} \lambda+O\left(\lambda^{2}\right)
$$

and

$$
a_{1}=\left[\widehat{v}(0)-\widehat{v}\left(2 p_{F}\right)\right] /\left(\pi \sin p_{F}\right)
$$

Note that while the exponent of the oscillating part is anomalous, the exponent of the non oscillating part is equal to the non interacting one, as a consequence of the vanishing of the Beta function (0.89); note also that the above result is in agrement reduces to $(0.47)$ when $\widehat{v}(k)=e^{i k}$ $p_{F}=\pi / 2$, in agreement with the exact solution of the $X X Z$ model. Finally the Cooper pair density correlation, that is the correlation of the operator $\rho_{\mathbf{x}}^{c}=a_{\mathbf{x}}^{+} a_{\mathbf{x}^{\prime}}^{+}+a_{\mathbf{x}}^{-} a_{\mathbf{x}^{\prime}}^{-}, \mathbf{x}^{\prime}=\left(x+1, x_{0}\right)$, behaves as

$$
\left\langle\rho_{\mathbf{x}}^{c} \rho_{\mathbf{0}}^{c}\right\rangle_{T} \sim \frac{1+A_{3}(\mathbf{x})}{2 \pi^{2}\left(x^{2}+v_{F}^{2} x_{0}^{2}\right)^{X_{-}}},
$$

with $X_{-}=1+a_{1} \lambda+O\left(\lambda^{2}\right), a_{1}$ being the same constant appearing in the first order of $X_{+}$.

The above formulas give a rather explicit expression for the correlations can be found. They were derived assuming the vanishing of the beta function $(0.84),(0.89)$, whose proof will be recalled below.

\subsection{Emerging symmetries and vanishing of Beta function}

The above Renormalization Group analysis relies on two non perturbative properties, namely (0.84) and (0.89); they can be checked at lowest orders in the renormalized expansion, but the cancellations are so complex that an explicit check at all orders is essentially impossible. The cancellations expressed by (0.84) and (0.89) are related to gauge symmetries which are however only asymptotic and not exact in the model (0.34). The key observation is to introduce a continuum model, related to the scaling limit of the 
lattice model (0.34), regularized by a non local interaction and an ultraviolet $\gamma^{N}, N>>1$ and infrared $\gamma^{h}, h<<0$ cut-offs, to be finally removed. The beta function of this continuum model coincides, up to asymptotically vanishing $O\left(\lambda \gamma^{j}\right)$ terms, with the one of the model (0.34); we will then show that gauge symmetries imply the vanishing of the Beta function of this model and consequently of the model (0.34). This strategy provides a rigorous way of implementing the ideas of emerging symmetries in the model (0.34).

We consider the following Grassman integral, which is the generating function of a system of massless Dirac fermions in the continuum with a non local current-current interaction.

$$
e^{W(J, \phi)}=\int P(d \psi) e^{V^{(N)}(\psi)+\int d \mathbf{x} J_{\mu, \mathbf{x}} j_{\mu, \mathbf{x}}+\sum_{\varepsilon= \pm}\left[\psi_{\mathbf{x}, \varepsilon}^{+} \phi_{\mathbf{x}, \varepsilon}^{-}+\psi_{\mathbf{x}, \varepsilon}^{-} \phi_{\mathbf{x}, \varepsilon}^{+}\right]}
$$

with $\mathbf{x}=\left(x_{0}, x\right) \in \mathbb{R}^{2}, \psi, \bar{\psi}$ are Euclidean $d=1+1$ spinors, $\bar{\psi}=\psi^{+} \gamma_{0}$, $\psi^{ \pm}=\left(\psi_{+}^{ \pm}, \psi_{-}^{ \pm}\right), P\left(d \psi^{(\leq N)}\right)$ is the fermionic gaussian integration with propagator

$$
g^{(h, N)}(\mathbf{k})=i \frac{\chi_{h, N}(\mathbf{k})}{\not \mathbf{k}}
$$

$\mathbf{k}=\gamma_{0} k_{0}+c \gamma_{1} k_{1}$, which in components appear to be equal to $g_{T, \varepsilon}^{(\leq N)}(\mathbf{x})$ $(0.81)$, and

$$
V^{(N)}(\psi)=\frac{1}{4} \widetilde{\lambda}_{\infty} \int d \mathbf{x} d \mathbf{y} v(\mathbf{x}-\mathbf{y}) j_{\mu}(\mathbf{x}) j_{\mu}(\mathbf{y})
$$

with $j_{\mu}(\mathbf{x})=\bar{\psi}_{\mathbf{x}} \gamma_{\mu} \psi_{\mathbf{x}}$ and $v(\mathbf{x}-\mathbf{y})$ a short range symmetric interaction $\widehat{v}(0)=1$; moreover

$$
\gamma_{0}=\left(\begin{array}{ll}
0 & 1 \\
1 & 0
\end{array}\right), \quad \gamma_{1}=\left(\begin{array}{cc}
0 & -i \\
i & 0
\end{array}\right)
$$

Finally the cut-off function $\chi_{h, N}(\mathbf{k})$ is $=1$ for $\gamma^{h-1} \leq \sqrt{k_{0}^{2}+c^{2} k^{2}} \leq$ $\gamma^{N+1}$ and zero otherwise (see Fig. 0.4). $\gamma^{N}$ plays the role of an ultraviolet cut-off, and at the end the limit $N \rightarrow \infty$ must be taken, while $\gamma^{h}$ acts as an infrared cut-off. Note that the propagator of the $\psi_{\varepsilon}$ fields is

$$
g_{\varepsilon}^{(h, N)}(\mathbf{k})=\frac{\chi_{h, N}(\mathbf{k})}{-i k_{0}+c \varepsilon k}=\frac{\chi_{h, N}(\mathbf{k})}{D_{\varepsilon}(\mathbf{k})}
$$

where $D_{\varepsilon}(\mathbf{k})=-i k_{0}+\varepsilon c k$ and the interaction is

$$
V^{(N)}(\psi)=\widetilde{\lambda}_{\infty} \int d \mathbf{x} d \mathbf{y} v(\mathbf{x}-\mathbf{y}) \psi_{+, \mathbf{x}}^{+} \psi_{+, \mathbf{x}}^{-} \psi_{-, \mathbf{y}}^{+} \psi_{-, \mathbf{y}}^{-}
$$




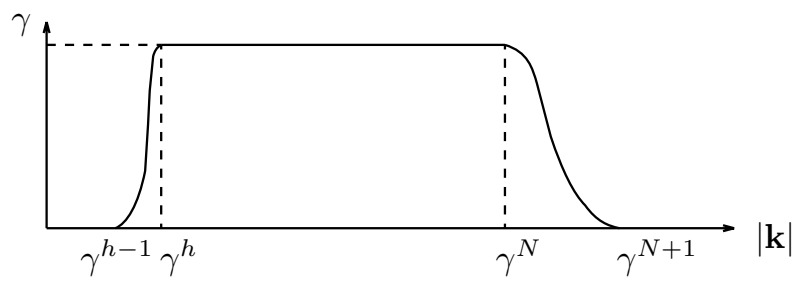

Fig. 0.4 The cut-off function $\chi_{h, N}(\mathbf{k})$

Therefore if we replace $v(\mathbf{x}-\mathbf{y})$ with $v(x-y) \delta\left(x_{0}-y_{0}\right)$ and we replace $\chi_{N}(\mathbf{k})$ with $\chi_{h, N}(k)$ we obtain, in the limit $-h, N \rightarrow \infty$, the Luttinger model.

Contrary to what happens in the model $(0.34)$, in which the lattice imposes an ultraviolet cut-off, the model (0.101) poses either an infrared and an ultraviolet problem. The fields is written as $\psi=\sum_{j=h}^{N} \psi^{(j)}$, where $\psi^{(j)}$ has propagator with support in $\gamma^{j-1} \leq \sqrt{k_{0}^{2}+c^{2} k^{2}} \leq \gamma^{j+1}$. According to power counting based on dimensional considerations, also in the ultraviolet region $(h>0)$ the scaling dimension is $2-n / 2-m$, but one can take advantage from the non-locality of the potential to improve the scaling dimension and show that indeed the dimension is always negative; in other words the theory is superrenormalizable in the ultraviolet while is renormalizable in the infrared region. The integration of the ultraviolet region is discussed in detail in $[34 ; 35]$ and is related to a previous analysis in [29] for the Yukawa 2 model. The main idea of the power counting improvement can be grasped from the following picture Fig. 0.5. Let us consider the kernels $W_{2,1}^{(k)}$ with $k>0$, with one external fields $J$ and two fermions fields $\psi$; the scaling dimension is apparently vanishing by power counting. Such kernels can be decomposed (without loosing the determinant bounds which are essential for convergence) in a sum of two kind of terms, as shown in Fig. 0.5.

Note that the scaling dimension $2-n / 2-m$ in the ultraviolet region is obtained integration over all the interactions $v(\mathbf{x}-\mathbf{y})$; however the terms represented by the second graph in Fig. 0.4 remain connected cutting the wiggly line representing the non local interaction $v(\mathbf{x}-\mathbf{y})$, so that we can simply bound $v$ by a constant and use one of the two propagators to perform the integral over the coordinates; this gives a gain $\gamma^{-2 k}$ in the bound, so that the dimension becomes negative for such terms. More explicitly, the 


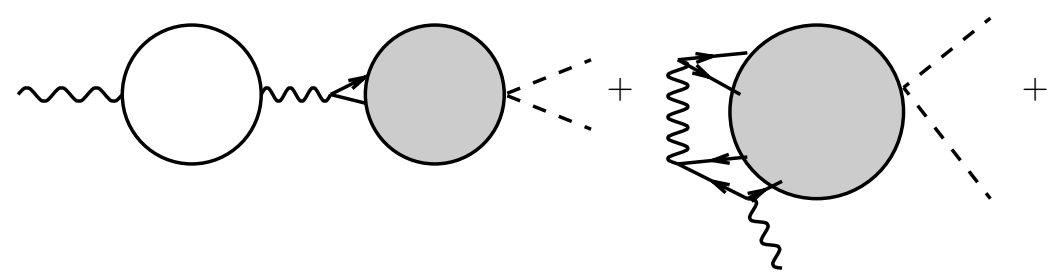

Fig. 0.5 Decomposition of $W_{2,1}^{(k)}$ : the gray blobs represent respectively $W_{2,1}^{(k)}$ and $W_{2,2}^{(k)}$, the paired wiggly lines represent $v$, the paired line $g^{(k, N)}$

second term in Fig. 0.4 can be bounded by

$$
C\left|\widetilde{\lambda}_{\infty}\right||v|_{L^{\infty}}\left|W_{2,2}^{(k)}\right|_{L^{1}} \sum_{k \leq i^{\prime} \leq j \leq i \leq N}\left|g^{(j)}\right|_{L^{1}}\left|g^{(i)}\right|_{L^{1}}\left|g^{\left(i^{\prime}\right)}\right|_{L^{\infty}} \leq C \widetilde{\lambda}_{\infty}^{2} \gamma^{-2 k}
$$

On the other hand the first kind of terms in Fig. 0.4 becomes disconnected cutting the internal wiggly line; however the local part of the first term is vanishing by symmetry as

$$
\int d \mathbf{x}\left[g_{\varepsilon}^{(k, N)}(\mathbf{x}-\mathbf{z})\right]^{2}=0
$$

so that the bound in the first term has an extra $\gamma^{-k}$. A similar power counting improvement can be repeated for the other terms to show that the dimension is always negative in the ultraviolet region. Note that in this analysis is essential that the interaction is short ranged, and it cannot be repeated for a local interaction in which $|v|_{L^{\infty}}$ is not bounded.

After the integration of the ultraviolet fields $\psi^{(N)}, \psi^{(N-1)}, \ldots, \psi^{(0)}$, we get a Grassman integral very similar to (0.67), with the difference that Grassman fields have propagators $g_{T, \varepsilon}^{(h, 0)}(\mathbf{x}-\mathbf{y})(0.81)$ with $c$ replacing $v_{F}$; the integration of the infrared scales is similar to the one for the model (0.34) but by symmetry $\nu_{j}=\delta_{j}=0, \widetilde{Z}_{j}^{(1)}=\widetilde{Z}_{j}^{(2)}$ and the single scale propagator is given by $g_{T, \varepsilon}^{(h)}(\mathbf{x}-\mathbf{y})$ with $v_{F}$ replaced by $c$. In the chain model, as we have seen, Lorentz symmetry is broken so that $\delta_{j} \neq 0$ (the velocity is renormalized) and $Z_{h-1}^{(1)} / Z_{h-1}^{(2)}=1+O(\lambda)$ ( $\rho$ and $J$ are not component of the relativistic current). The effective coupling verify a flow equation

$$
\widetilde{\lambda}_{j-1}=\widetilde{\lambda}_{j}+\bar{\beta}_{\lambda}^{(j)}\left(\widetilde{\lambda}_{j}, \ldots \widetilde{\lambda}_{0}\right)+O\left(\widetilde{\lambda}_{\infty}^{2} \gamma^{\vartheta j}\right)
$$

where $\bar{\beta}_{\lambda}^{(j)}\left(\tilde{\lambda}_{j}, \ldots \tilde{\lambda}_{j}\right)$ is the same as function of $\tilde{\lambda}_{j}$ of the one appearing in (0.83) for the model (0.101), provided that we choose $c=v_{F}$; the same is 
true for $\bar{\beta}_{1}^{(j)}(0.88)$. Moreover

$$
\widetilde{Z}_{j} \sim \gamma^{\eta j} \quad \eta=\log _{\gamma}\left(1+\bar{\beta}_{z}^{(-\infty)}\left(\tilde{\lambda}_{-\infty}, \ldots, \tilde{\lambda}_{-\infty}\right)\right)
$$

where $\bar{\beta}_{z}^{(-\infty)}$ is the same as appearing in (0.86)

We have then seen that the dominant part of the beta function for the effective coupling $\widetilde{\lambda}_{j}$ and for $\widetilde{Z}_{j}^{1} / \widetilde{Z}_{j}$ coincides with the one of the chain model (0.34). We will take now advantage from the fact that the model (0.101) verify extra symmetries, which at the end will lead to the proof of $(0.84)$ and (0.89). Note first that, denoting $\langle\ldots\rangle_{h, N}$ the averages in the model (0.101) with infrared cut-off $\gamma^{h}$ and ultraviolet cut-off $\gamma^{N}$, for momenta $\mathbf{k} \sim \gamma^{h}$ we get, if $D_{\varepsilon}(\mathbf{k})=-i k_{0}+\varepsilon c k$

$$
\begin{aligned}
& \left\langle\widehat{\rho}_{\mathbf{p}} \widehat{\psi}_{2 \mathbf{k}, \varepsilon}^{-} \widehat{\psi}_{-\mathbf{k}, \varepsilon}^{+}\right\rangle_{h, N}=-\frac{\widetilde{Z}_{h}^{(1)}}{\widetilde{Z}_{h}^{2} D_{\varepsilon}(\mathbf{k})^{2}}\left(1+O\left(\widetilde{\lambda}_{h}^{2}\right)\right) \\
& \left\langle\widehat{\psi}_{\mathbf{k}, \varepsilon}^{-} \widehat{\psi}_{\mathbf{k}, \varepsilon}^{+}\right\rangle_{h, N}=\frac{1}{\widetilde{Z}_{h} D_{\varepsilon}(\mathbf{k})}\left(1+O\left(\widetilde{\lambda}_{h}^{2}\right)\right) \\
& \left\langle\widehat{\psi}_{\mathbf{k},+}^{-} \widehat{\psi}_{-\mathbf{k},+}^{+} \widehat{\psi}_{\mathbf{k},-}^{-} \widehat{\psi}_{-\mathbf{k},-}^{+}\right\rangle_{h, N}=\frac{-\widetilde{\lambda}_{h}+O\left(\widetilde{\lambda}_{h}^{2}\right)}{\widetilde{Z}_{h}^{2} D_{+}(\mathbf{k})^{2} D_{-}(\mathbf{k})^{2}}
\end{aligned}
$$

From the above equations we see that the value of the effective renormalizations $\widetilde{Z}_{h}^{(1)}, \widetilde{Z}_{h}$ and of the effective coupling $\widetilde{\lambda}_{h}$ are related to the value of the two, three and four point functions with an infrared scale $\gamma^{h}$, computed for momenta close to the infrared cut-off scale. On the other hand, the value of $\widetilde{Z}_{k}^{(1)}, \widetilde{Z}_{k}, \widetilde{\lambda}_{k}$ for $k \geq h$ in a theory with infrared cut-off at scale $\gamma^{h}$ are the same as in a theory with no infrared cut-off, as a consequence of the compact support properties of the single scale propagators. Therefore, we can consider a sequence of models varying the value of the infrared cut-off scale; if we can derive a set of Ward Identities relating the correlations and we compute them at the infrared scale, we get, from $(0.110),(0.111),(0.112)$, relations between the effective renormalizations and couplings. In order to do that one has to face a well known problem, which is present in any Wilsonian RG approach; the momentum cut-off breaks the local symmetries and produces additional terms in the Ward identities.

In order to understand the idea let us start considering the functional integral (0.101) in which the cut-off function $\chi_{h, N}$ is replaced by 1 ; the Grassmann integral is therefore only formal (to be well defined it requires a regularization) but let us for the moment ignore this fact. We can now perform the change of variables

$$
\psi_{\mathbf{x}, \varepsilon}^{ \pm} \rightarrow e^{ \pm i \alpha_{\varepsilon, \mathbf{x}}} \psi_{\mathbf{x}, \varepsilon}^{ \pm}
$$


and perform a derivative with respect to $\alpha_{\mathbf{x}, \varepsilon}$ and to the external fields we get the following Ward Identity, if $D_{\varepsilon}(\mathbf{p})=-i p_{0}+\varepsilon c p$

$$
D_{\varepsilon}(\mathbf{p})\left\langle\widehat{\rho}_{\mathbf{p}, \varepsilon} \widehat{\psi}_{\mathbf{k}-\mathbf{p}, \varepsilon^{\prime}}^{-} \widehat{\psi}_{\mathbf{k}, \varepsilon^{\prime}}^{+}\right\rangle=\delta_{\varepsilon, \varepsilon^{\prime}}\left[\left\langle\widehat{\psi}_{\mathbf{k}-\mathbf{p}, \varepsilon^{\prime}}^{-} \widehat{\psi}_{\mathbf{k}-\mathbf{p}, \varepsilon^{\prime}}^{+}\right\rangle-\left\langle\widehat{\psi}_{\mathbf{k}, \varepsilon^{\prime}}^{-} \widehat{\psi}_{\mathbf{k}, \varepsilon^{\prime}}^{+}\right\rangle\right]
$$

This relation says that the two and three point function are not independent and it suggests, using $(0.110),(0.111)$, that $\widetilde{Z}_{h}^{(1)} \sim \widetilde{Z}_{h}$, that is the current renormalization and the wave function renormalization diverges with the same exponent. However, the above Ward Identity is not true in presence of the cut-off function $\chi_{h, N}$.

If we repeat the above computation in presence of the cut-off function $\chi_{h, N}$ we get that the Ward Identity acquires a correction term; the true Ward Identity for the model (0.101) is

$$
\begin{aligned}
& D_{\varepsilon}(\mathbf{p})\left\langle\widehat{\rho}_{\mathbf{p}, \varepsilon} \widehat{\psi}_{\mathbf{k}-\mathbf{p}, \varepsilon^{\prime}}^{-} \widehat{\psi}_{\mathbf{k}, \varepsilon^{\prime}}^{+}\right\rangle_{h, N}= \\
& \delta_{\varepsilon, \varepsilon^{\prime}}\left[\left\langle\widehat{\psi}_{\mathbf{k}-\mathbf{p}, \varepsilon^{\prime}}^{-} \widehat{\psi}_{\mathbf{k}-\mathbf{p}, \varepsilon^{\prime}}^{+}\right\rangle_{h, N}-\left\langle\widehat{\psi}_{\mathbf{k}, \varepsilon^{\prime}}^{-} \widehat{\psi}_{\mathbf{k}, \varepsilon^{\prime}}^{+}\right\rangle_{h, N}\right]+\widehat{\Delta}_{\varepsilon, \varepsilon^{\prime}}(\mathbf{p}, \mathbf{k})
\end{aligned}
$$

with

$$
\widehat{\Delta}_{\varepsilon, \varepsilon^{\prime}}(\mathbf{p}, \mathbf{k})=\frac{1}{L^{2}} \sum_{\mathbf{k}^{\prime}} C_{h, N}\left(\mathbf{k}^{\prime}, \mathbf{k}^{\prime}-\mathbf{p}\right)<\widehat{\psi}_{\mathbf{k}^{\prime}, \varepsilon}^{+} \widehat{\psi}_{\mathbf{k}^{\prime}-\mathbf{p}, \varepsilon}^{-} ; \widehat{\psi}_{\mathbf{k}, \varepsilon^{\prime}}^{-} \widehat{\psi}_{\mathbf{k}-\mathbf{p}, \varepsilon^{\prime}}^{+}>
$$

where

$$
C_{h, N}(\mathbf{k}, \mathbf{k}-\mathbf{p})=\left(\chi_{h, N}^{-1}(\mathbf{k}-\mathbf{p})-1\right) D_{\varepsilon}(\mathbf{k}-\mathbf{p})-\left(\chi_{h, N}^{-1}(\mathbf{k})-1\right) D_{\varepsilon}(\mathbf{k})
$$

With respect to the (0.114), the presence of the infrared and ultraviolet cut

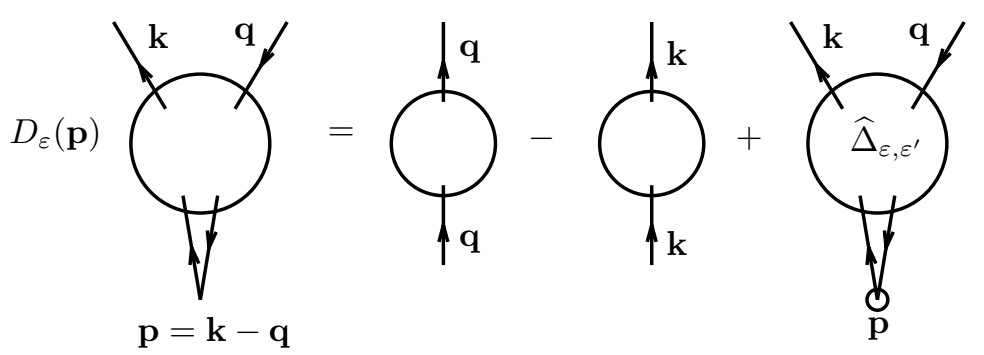

Fig. 0.6 Graphical representation of the Ward identity (0.115) 
off produce the extra term $\Delta_{\varepsilon \varepsilon^{\prime}}(\mathbf{p}, \mathbf{k})$ in $(0.115)$; its presence can be easily checked at lowest order in perturbation theory starting from the following (trivial) identity for the propagator (0.104)

$$
\begin{aligned}
& D_{\varepsilon}(\mathbf{p}) \frac{\chi_{h, N}(\mathbf{k})}{D_{\varepsilon}(\mathbf{k})} \frac{\chi_{h, N}(\mathbf{k}-\mathbf{p})}{D_{\varepsilon}(\mathbf{k}-\mathbf{p})}=\frac{\chi_{h, N}(\mathbf{k}-\mathbf{p})}{D_{\varepsilon}(\mathbf{k}-\mathbf{p})}-\frac{\chi_{h, N}(\mathbf{k})}{D_{\varepsilon}(\mathbf{k})}+ \\
& C_{h, N}(\mathbf{k}, \mathbf{k}-\mathbf{p}) \frac{\chi_{h, N}(\mathbf{k})}{D_{\varepsilon}(\mathbf{k})} \frac{\chi_{h, N}(\mathbf{k}-\mathbf{p})}{D_{\varepsilon}(\mathbf{k}-\mathbf{p})}
\end{aligned}
$$

Following the previous formal computation, one could be tempted to conclude that $\Delta_{\varepsilon, \varepsilon^{\prime}}$ is vanishing in the limit $-h, N \rightarrow \infty$; this conclusion is however false and the following formula holds, proved in [34; $35]$

$$
\begin{aligned}
& \Delta_{\varepsilon, \varepsilon^{\prime}}(\mathbf{p}, \mathbf{k})= \\
& \frac{\widetilde{\lambda}_{\infty}}{4 \pi c} v(\mathbf{p}) D_{-\varepsilon}(\mathbf{p})\left\langle\widehat{\rho}_{\mathbf{p},-\varepsilon} \widehat{\psi}_{\mathbf{k}-\mathbf{p}, \varepsilon^{\prime}}^{-} \widehat{\psi}_{\mathbf{k}, \varepsilon^{\prime}}^{+}\right\rangle_{h, N}+D_{\varepsilon}(\mathbf{p}) R_{\varepsilon, \varepsilon^{\prime}}(\mathbf{p} ; \mathbf{k})
\end{aligned}
$$

and, for $h \leq 0, N$ large enough and $|\mathbf{p}|,|\mathbf{k}| \sim \gamma^{h}$

$$
\left|R_{\varepsilon, \varepsilon^{\prime}}(\mathbf{p} ; \mathbf{k})\right| \leq C\left|\tilde{\lambda}_{\infty}\right| \frac{\gamma^{-2 h}}{Z_{h}}
$$

Again the proof of (0.120) is technical, but the main ideas can be understood qualitatively. The term $\Delta_{\varepsilon, \varepsilon^{\prime}}$ can be written as the derivative of a Grasmman integral, similar to (0.101) but in which $\int d \mathbf{x} J_{\mu, \mathbf{x}} j_{\mu, \mathbf{x}}$ is replaced by

$$
\int d \mathbf{k} d \mathbf{p} J_{\mathbf{p}} C_{h, N}(\mathbf{k}, \mathbf{k}+\mathbf{p}) \widehat{\psi}_{\mathbf{k}, \varepsilon}^{+} \widehat{\psi}_{\mathbf{k}+\mathbf{p}, \varepsilon}^{-}
$$

The analysis of the ultraviolet scales is similar to the one of (0.101); the

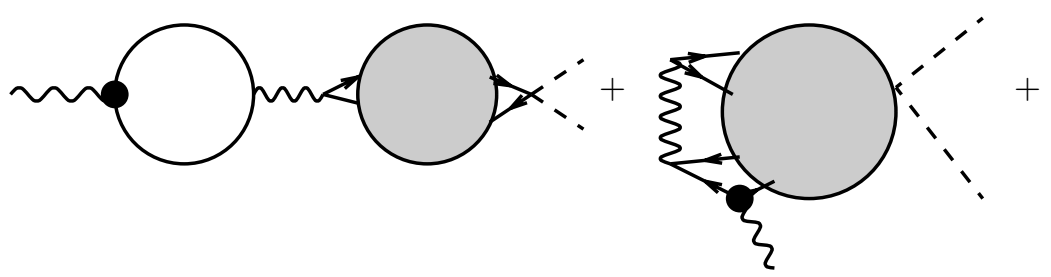

Fig. 0.7 Decomposition of $\widetilde{W}_{1,2}^{(k)}$

terms with two external fermionic fields and a $J$ external field $\widetilde{W}_{2,1}^{(h)}$ are 
again dimensionally marginal and one needs a dimensional improvement produced by the decomposition. The second term in Fig. 0.7 represents the terms which remain connected cutting the wiggly line representing the non local interaction $v(\mathbf{x}-\mathbf{y})$, so that we can simply bound $v$ by a constant and use one of the two propagators to perform the integral over the coordinates; this gives a gain $\gamma^{-2 k}$ in the bound, so that the dimension becomes negative for such terms. There is however an extra gain due to the fact that necessarily at least one of the two fields in (0.121) must have scale $N$; this is a consequence of the definition (0.117) $\left(1-\chi_{N}\right.$ has to be non vanishing). Therefore, the second term in Fig. 0.7 can be bounded by

$$
C\left|\widetilde{\lambda}_{\infty}\right||v|_{L^{\infty}}\left|\widetilde{W}_{2,2}^{(k)}\right|_{L^{1}} \sum_{k \leq i^{\prime} \leq j \leq i \leq N}\left|g^{(N)}\right|_{L^{1}}\left|g^{(i)}\right|_{L^{1}}\left|g^{\left(i^{\prime}\right)}\right|_{L^{\infty}} \leq C \widetilde{\lambda}_{\infty}^{2} \gamma^{-N-k}
$$

so that its contribution is now vanishing as $N \rightarrow \infty$. Another crucial difference with respect to the analysis in (0.4) is the fact that the local part of the bubble in the first term is non vanishing and given by

$$
\begin{aligned}
& \left.\lim _{N \rightarrow \infty} \widetilde{\lambda}_{\infty} \int \frac{d \mathbf{k}}{(2 \pi)^{2}} C_{N}(\mathbf{k}, \mathbf{k}-\mathbf{p}) g_{\varepsilon}^{(\leq N)}(\mathbf{k}) g_{\varepsilon}^{(\leq N)}(\mathbf{k}-\mathbf{p})\right|_{\mathbf{p}=0} \\
& =-\frac{\widetilde{\lambda}_{\infty}}{4 \pi c} D_{-\varepsilon}(\mathbf{p}) \int_{0}^{\infty} d \rho \chi_{0}^{\prime}(\rho)=\frac{\widetilde{\lambda}_{\infty}}{4 \pi c} D_{-\varepsilon}(\mathbf{p})
\end{aligned}
$$

This explain the presence of the first term of the r.h.s. of (0.120).

By inserting (0.120) in (0.115), computing the resulting expression at the cut-off scale $\gamma^{h}$ and using (0.110)a and (0.111) we get that

$$
\frac{\widetilde{Z}_{h}^{(1)}}{\widetilde{Z}_{h}}=1+O\left(\bar{\lambda}_{h}^{2}\right)
$$

Comparing this with (0.88) we get, by a contradiction argument, the asymptotic vanishing of the beta function (0.89).

A similar argument can be repeated for proving (0.84). One writes a Schwinger-Dyson equation for the model (0.101), that is

$$
\begin{aligned}
& -\left\langle\psi_{\mathbf{k}_{1},+}^{-} \psi_{\mathbf{k}_{2},+}^{+} \psi_{\mathbf{k}_{3},-}^{-} \psi_{\mathbf{k}_{4},-}^{+}\right\rangle_{h, N}=\widetilde{\lambda}_{\infty} \widehat{g}_{-}^{(h, N)}\left(\mathbf{k}_{4}\right)\left[v\left(\mathbf{k}_{1}-\mathbf{k}_{2}\right)\right. \\
& \left\langle\psi_{\mathbf{k}_{1},+}^{-} \psi_{\mathbf{k}_{2},+}^{+} \rho_{\mathbf{k}_{1}-\mathbf{k}_{2},+}\right\rangle_{h, N}\left\langle\psi_{\mathbf{k}_{3},-}^{-} \psi_{\mathbf{k}_{3},-}^{+}\right\rangle_{h, N} \\
& \left.+\frac{1}{L^{2}} \sum_{\mathbf{p}} \widetilde{\lambda}_{\infty} \widehat{v}(\mathbf{p})\left\langle\rho_{\mathbf{p},+} \psi_{\mathbf{k}_{1},+}^{-} \psi_{\mathbf{k}_{2},+}^{+} \psi_{\mathbf{k}_{3},-}^{-} \psi_{\mathbf{k}_{4}-\mathbf{p},-}^{+}\right\rangle_{h, N}\right]
\end{aligned}
$$

The 1.h.s is proportional to $\lambda_{h}$ when $\mathbf{k}_{i} \sim \gamma^{h}$ by (0.112) and one writes in the first term in the r.h.s. the function $\left\langle\psi_{\mathbf{k}_{1},+}^{-} \psi_{\mathbf{k}_{2},+}^{+} \rho_{\mathbf{k}_{1}-\mathbf{k}_{2},+}\right\rangle$ in terms 
of the 2-point function by using the Ward Identity (0.120) and (0.115). Similarly we can use for the second term in the r.h.s. a Ward identity for $\left\langle\rho_{\mathbf{p},+} \psi_{\mathbf{k}_{1},+}^{-} \psi_{\mathbf{k}_{2},+}^{+} \psi_{\mathbf{k}_{3},-}^{-} \psi_{\mathbf{k}_{4}-\mathbf{p},-}^{+}\right\rangle_{h, N}$; now one gets a term involving the integral of the correction $R$ in the Ward Identity and the main technical problem, discussed in [33],[42], is to bound such a term uniformly in $h$; the conclusion is that the r.h.s. is proportional to $\widetilde{\lambda}_{\infty}+O\left(\widetilde{\lambda}_{\infty}^{2}\right)$ and this implies the vanishing of the beta function in (0.108) and consequently (0.84); therefore $\widetilde{\lambda}_{-\infty}$ is analytic in $\widetilde{\lambda}_{\infty}$ and

$$
\widetilde{\lambda}_{-\infty}=\widetilde{\lambda}_{\infty}+O\left(\widetilde{\lambda}_{\infty}^{2}\right)
$$

\subsection{Ward Identities and anomalies}

In the previous section we have considered a fermionic model (0.101) with linear dispersion relation, a momentum infrared and ultraviolet cut-off and a non-local interaction; the infrared behavior of this model is the same as the one of the chain model (0.34) but it verifies extra symmetries allowing to deduce Ward Identities. The momentum cut-off produces corrections with respect to the naive Ward Identities which are not small at all, and are not vanishing even when cut-offs are removed. Indeed (0.115) and (0.120) acquire a simpler form in the limit $-h, N \rightarrow \infty$ at fixed momenta where they can be rewritten as

$$
\begin{aligned}
& D_{\varepsilon}(\mathbf{p})\left\langle\widehat{\rho}_{\mathbf{p}, \varepsilon} \widehat{\psi}_{\mathbf{k}-\mathbf{p}, \varepsilon}^{-} \widehat{\psi}_{\mathbf{k}, \varepsilon}^{+}\right\rangle= \\
& \left\langle\widehat{\psi}_{\mathbf{k}-\mathbf{p}, \varepsilon}^{-} \widehat{\psi}_{\mathbf{k}-\mathbf{p}, \varepsilon}^{+}\right\rangle-\left\langle\widehat{\psi}_{\mathbf{k}, \varepsilon}^{-} \widehat{\psi}_{\mathbf{k}, \varepsilon}^{+}\right\rangle+\frac{\widetilde{\lambda}_{\infty}}{4 \pi c} D_{-\varepsilon}(\mathbf{p})\left\langle\widehat{\rho}_{\mathbf{p},-\varepsilon} \widehat{\psi}_{\mathbf{k}-\mathbf{p}, \varepsilon}^{-} \widehat{\psi}_{\mathbf{k}, \varepsilon}^{+}\right\rangle \\
& D_{-\varepsilon}(\mathbf{p})\left\langle\widehat{\rho}_{\mathbf{p},-\varepsilon} \widehat{\psi}_{\mathbf{k}-\mathbf{p}, \varepsilon}^{-} \widehat{\psi}_{\mathbf{k}, \varepsilon}^{+}\right\rangle=\frac{\widetilde{\lambda}_{\infty}}{4 \pi c} D_{\varepsilon}(\mathbf{p})\left\langle\widehat{\rho}_{\mathbf{p}, \varepsilon} \widehat{\psi}_{\mathbf{k}-\mathbf{p}, \varepsilon}^{-} \widehat{\psi}_{\mathbf{k}, \varepsilon}^{+}\right\rangle
\end{aligned}
$$

or, using the relativistic notation $j_{\mu}=\bar{\psi} \gamma_{\mu} \psi j_{\mu, 5}=\bar{\psi} \gamma_{\mu} \gamma_{5} \psi$ and using that $j_{0}=-i j_{5,1}=\rho_{1}+\rho_{-1}, j_{1}=i j_{5,0}=i\left(\rho_{1}-\rho_{-1}\right)$ and calling $\mathbf{p}_{\mu}=(\omega, c p)$

$$
\begin{aligned}
& -i \mathbf{p}_{\mu}<j_{\mu, \mathbf{p}} \psi_{\mathbf{k}} \psi_{\mathbf{k}+\mathbf{p}}^{+}>=A\left[<\psi_{\mathbf{k}} \psi_{\mathbf{k}}^{+}>-<\psi_{\mathbf{k}+\mathbf{p}} \psi_{\mathbf{k}+\mathbf{p}}^{+}>\right] \\
& -i \mathbf{p}_{\mu}<j_{5, \mu, \mathbf{p}} \psi_{\mathbf{k}} \psi_{\mathbf{k}+\mathbf{p}}^{+}>=\bar{A} \gamma_{5}\left[<\psi_{\mathbf{k}} \psi_{\mathbf{k}}^{+}>-<\psi_{\mathbf{k}+\mathbf{p}} \psi_{\mathbf{k}+\mathbf{p}}^{+}>\right]
\end{aligned}
$$

with

$$
A(\mathbf{p})=\frac{1}{1-\tau \widehat{v}(\mathbf{p})} \quad \bar{A}(\mathbf{p})=\frac{1}{1+\tau \widehat{v}(\mathbf{p})}
$$

and

$$
\tau=\frac{\widetilde{\lambda}_{\infty}}{4 \pi c}
$$


In the same way the Ward Identities for the density operators in the model (0.101) are, in the limit of removed cut-off $-h, N \rightarrow \infty$

$$
\begin{aligned}
& D_{+}(\mathbf{p})<\rho_{\mathbf{p},+} \rho_{-\mathbf{p},+}>-\tau D_{-}(\mathbf{p})<\rho_{\mathbf{p},-} \rho_{-\mathbf{p},+}>=\frac{1}{4 \pi c} D_{-}(\mathbf{p}) \\
& D_{-}(\mathbf{p})<\rho_{\mathbf{p},-} \rho_{-\mathbf{p},+}>-\tau D_{+}(\mathbf{p})<\rho_{\mathbf{p},+} \rho_{-\mathbf{p},+}>=0
\end{aligned}
$$

from which we get

$$
\begin{aligned}
& <j_{\mathbf{p}}^{(0))} j_{-\mathbf{p}}^{(0)}>=-\frac{1}{4 \pi c} \frac{1}{1-\tau^{2}}\left[\frac{D_{-}(\mathbf{p})}{D_{+}(\mathbf{p})}+\frac{D_{+}(\mathbf{p})}{D_{-}(\mathbf{p})}-2 \tau\right] \\
& <j_{\mathbf{p}}^{(1))} j_{-\mathbf{p}}^{(1)}>=-\frac{1}{4 \pi c} \frac{1}{1-\tau^{2}}\left[\frac{D_{-}(\mathbf{p})}{D_{+}(\mathbf{p})}+\frac{D_{+}(\mathbf{p})}{D_{-}(\mathbf{p})}+2 \tau\right]
\end{aligned}
$$

Finally we can write the Schwinger-function for the 2-point function in the model $(0.101)$

$$
\begin{aligned}
& \left\langle\psi_{\mathbf{k}, \varepsilon}^{-} \psi_{\mathbf{k}, \varepsilon}^{+}\right\rangle_{h, N}=g_{\omega}^{[h, N]}(\mathbf{k})+ \\
& \left.\widetilde{\lambda}_{\infty} g_{\varepsilon}^{[h, N]}(\mathbf{k}) \frac{1}{L^{2}} \sum_{\mathbf{p}} \widehat{v}(\mathbf{p})\left\langle\rho_{\mathbf{p},-\varepsilon} \psi_{\mathbf{k},-}^{-} \psi_{\mathbf{k}-\mathbf{p},-}^{+}\right\rangle_{h, N}\right]
\end{aligned}
$$

We can now insert in the last term the Ward Identity (0.115) and (0.120); it turns out that the integral of the correction is vanishing so that in the limit $-h, N \rightarrow \infty$

$$
D_{\varepsilon}(\mathbf{k})\left\langle\widehat{\psi}_{\mathbf{k}, \varepsilon}^{-} \widehat{\psi}_{\mathbf{k}, \varepsilon}^{+}\right\rangle=1+\widetilde{\lambda}_{\infty} \int \frac{d \mathbf{p}}{(2 \pi)^{2}}[A(\mathbf{p})-\bar{A}(\mathbf{p})] \frac{v(\mathbf{p})}{D_{-\varepsilon}(\mathbf{p})}\left\langle\widehat{\psi}_{\mathbf{k}-\mathbf{p}, \varepsilon}^{-} \widehat{\psi}_{\mathbf{k}-\mathbf{p}, \varepsilon}^{+}\right\rangle
$$

Passing in Fourier transform we get a PDE whose solution is given by

$$
\left\langle\psi_{\mathbf{x}}^{-} \psi_{\mathbf{y}}^{+}\right\rangle \sim g_{\varepsilon}(\mathbf{x}-\mathbf{y}) \frac{1}{|\mathbf{x}-\mathbf{y}|^{\eta}}
$$

with

$$
\eta=\frac{\tilde{\lambda}_{\infty}}{4 \pi c}\left[\frac{1}{1-\tau}-\frac{1}{1+\tau}\right]
$$

In the same way we can consider the limit of removed cut-off of (0.125) and we get

$$
\left\langle\psi_{\mathbf{x}, \varepsilon}^{+} \psi_{\mathbf{x},-\varepsilon}^{-} \psi_{\mathbf{y},-\varepsilon}^{+} \psi_{\mathbf{y}, \varepsilon}^{-}\right\rangle_{T} \sim \frac{C}{|\mathbf{x}-\mathbf{y}|^{2 X_{+}}}
$$

and

$$
\left\langle\psi_{\mathbf{x}, \varepsilon}^{+} \psi_{\mathbf{x},-\varepsilon}^{+} \psi_{\mathbf{y},-\varepsilon}^{-} \psi_{\mathbf{y}, \varepsilon}^{-}\right\rangle_{T} \sim \frac{C}{|\mathbf{x}-\mathbf{y}|^{2 X_{-}}}
$$


so that

$$
\begin{aligned}
& X_{+}=1-\bar{A}(0)\left(\lambda_{\infty} / 2 \pi c\right)=1-\frac{\left(\widetilde{\lambda}_{\infty} / 2 \pi c\right)}{1+\left(\widetilde{\lambda}_{\infty} / 4 \pi c\right)} \\
& X_{=1}+A(0)\left(\lambda_{\infty} / 2 \pi c\right)=1+\frac{\left(\widetilde{\lambda}_{\infty} / 2 \pi c\right)}{1-\left(\widetilde{\lambda}_{\infty} / 4 \pi c\right)} ;
\end{aligned}
$$

The exponents in the model (0.101) verify the relations $(0.48)$, even if the exponents are of course different with respect to the Luttinger model ones $(0.22)$.

The Grassmann integral (0.101) (with no infrared cut-off) can be rewritten, via an Hubbard-Stratonovich transformation, in the following way if $\widetilde{\lambda}_{\infty}=e^{2}$

$$
\begin{aligned}
& \mathcal{W}_{N}(J, \phi)= \\
& \log \int P\left(d \psi^{(\leq N)}\right) P(d A) e^{\int d \mathbf{x}\left[e \bar{\psi}_{\mathbf{x}}\left(A_{\mu, \mathbf{x}} \gamma_{\mu}\right) \psi_{\mathbf{x}}+J_{\mu, \mathbf{x}} A_{\mu, \mathbf{x}}+\phi_{\mathbf{x}} \bar{\psi}_{\mathbf{x}}+\bar{\phi}_{\mathbf{x}} \psi_{\mathbf{x}}\right]}
\end{aligned}
$$

in which the bosonic propagator is given by $\left\langle A_{\mu, \mathbf{x}} A_{\nu, \mathbf{y}}\right\rangle=\delta_{\mu, \nu} v(\mathbf{x}-\mathbf{y})$. Therefore, the model $(0.101)$ can be equivalently written as a Quantum Field Theory model in $d=1+1$ in which massless Dirac fermions interact with a vector boson field. The WI (0.130) can be rewritten as

$$
\begin{aligned}
& -i \gamma_{\mu} \mathbf{p}_{\mu}<j_{5, \mu, \mathbf{p}} \psi_{\mathbf{k}, \omega} \bar{\psi}_{\mathbf{k}+\mathbf{p}}>= \\
& {\left[<\psi_{\mathbf{k}} \bar{\psi}_{\mathbf{k}}>-<\psi_{\mathbf{k}+\mathbf{p}} \bar{\psi}_{\mathbf{k}+\mathbf{p}}^{-}>\right]+\frac{\tau}{e} \varepsilon_{\mu, \nu}<A_{\nu, \mathbf{p}} \psi_{\mathbf{k}, \omega} \bar{\psi}_{\mathbf{k}+\mathbf{p}}>}
\end{aligned}
$$

where we have used that

$$
\varepsilon_{\mu, \nu}<A_{\nu, \mathbf{p}} \psi_{\mathbf{k}, \omega} \bar{\psi}_{\mathbf{k}+\mathbf{p}}>=e v(\mathbf{p})<j_{5, \mu, \mathbf{p}} \psi_{\mathbf{k}, \omega} \bar{\psi}_{\mathbf{k}+\mathbf{p}}>
$$

where $\frac{\tau}{e}=\frac{e}{4 \pi c}$ is the chiral anomaly. The anomaly is linear in $e$, a property called anomaly non renormalization; it was first proved by Adler and Bardeen as an identity in perturbation theory [44] in QED $_{4}$ and (0.141) is the form it acquires in $d=1+1$, see [45]. The results in [44; 45] are however true order by order in the expansion, while (0.141) is a non perturbative statement (it makes no use of the loop cancellation or on any graphs arguments) obtained by a rigorous analysis of Grassmann integrals, and its validity relies on $(0.120)$.

The model (0.101) can be also considered a regularization of the massless Thirring model [4]; more exactly, if $v(x)$ is a regularized delta and $\lim _{K \rightarrow \infty} v(x)=\delta(x)$, the Thirring model can be constructed taking the limits $K, N \rightarrow \infty$. Such limit gives a non trivial limit only if 
we perform the change of variables $\psi \rightarrow \sqrt{Z} \psi$ and we choose $Z$ diverging in the limit $K, N \rightarrow \infty$. If the limit $K \rightarrow \infty$ is removed after the limit $N \rightarrow \infty$ one has to choose $Z=\gamma^{-\eta K}$ and it is found [34; $42]$ that the WI is given by $(0.129),(0.129),(0.130)$ with $v(p)$ replaced by 1 . This Ward Identity, obtained by the analysis of the Grassman integral (0.101), coincides with the one derived by Johnson [4] postulating the validity of anomalous commutation rules

$$
\left[\psi(x), j^{0}(y)\right]=a \delta(x-y) \psi(y) \quad\left[\psi(x), j_{5}^{0}(y)\right]=\bar{a} \delta(x-y) \psi(y)
$$

and fixing the value of $a, \bar{a}$ by a self consistence argument.

On the other hand, if one performs the limit in the opposite order, that is the $N \rightarrow \infty$ limit is taken after $K \rightarrow \infty$ choosing $Z=\gamma^{-\eta N}$ one finds again the Ward Identity (0.129), (0.129) but now $\tau$ is given by [43]

$$
\tau=\frac{\widetilde{\lambda}_{\infty}}{4 \pi c}+b \widetilde{\lambda}_{\infty}^{2}+O\left(\widetilde{\lambda}_{\infty}^{3}\right)
$$

that is the anomaly acquires higher orders corrections. The validity of the anomaly non renormalization in the Thirring model depends then on how the cut-offs are removed. Having higher orders correction in the anomaly would prevent to get simple expressions of the exponents like (0.137), (0.138) and would prevent the possibility of checking the scaling relations.

The Ward Identities (0.129) for the lattice model (0.34) are different with respect to the Luttinger model ones $(0.32),(0.33)$. However a Renormalization Group analysis similar to the one for the model (0.101) could be repeated also for the Luttinger model; in such case, the cut-off $\chi_{N}(\mathbf{k})$ should be replaced by a cut-off involving only the spatial momenta, that is $\chi_{N}(k)$ depending only from the spatial momentum. The ultraviolet fields can be integrated [46] and the Ward Identities acquire a correction given by $(0.115)$ with

$$
\lim _{-h, N \rightarrow \infty} \Delta_{\varepsilon, \varepsilon^{\prime}}(\mathbf{p} ; \mathbf{k})=\frac{\widetilde{\lambda}_{\infty}}{2 \pi} \widehat{v}(p) p\left\langle\rho_{\mathbf{p},-\varepsilon} \widehat{\psi}_{\mathbf{k}-\mathbf{p}, \varepsilon^{\prime}}^{-} \widehat{\psi}_{\mathbf{k}, \varepsilon^{\prime}}^{+}\right\rangle
$$

The computation is analogue to $(0.123)$ with $\chi_{N}(\mathbf{k})$ replaced by $\chi_{N}(k)$. Therefore $(0.127)$ is replaced by

$$
\begin{aligned}
& D_{\varepsilon}(\mathbf{p})\left\langle\widehat{\rho}_{\mathbf{p}, \varepsilon} \widehat{\psi}_{\mathbf{k}-\mathbf{p}, \varepsilon}^{-} \widehat{\psi}_{\mathbf{k}, \varepsilon}^{+}\right\rangle= \\
& \left\langle\widehat{\psi}_{\mathbf{k}-\mathbf{p}, \varepsilon}^{-} \widehat{\psi}_{\mathbf{k}-\mathbf{p}, \varepsilon}^{+}\right\rangle-\left\langle\widehat{\psi}_{\mathbf{k}, \varepsilon}^{-} \widehat{\psi}_{\mathbf{k}, \varepsilon}^{+}\right\rangle+\frac{\tilde{\lambda}_{\infty}}{2 \pi} p\left\langle\widehat{\rho}_{\mathbf{p},-\varepsilon} \widehat{\psi}_{\mathbf{k}-\mathbf{p}, \varepsilon}^{-} \widehat{\psi}_{\mathbf{k}, \varepsilon}^{+}\right\rangle \\
& D_{-\varepsilon}(\mathbf{p})\left\langle\widehat{\rho}_{\mathbf{p},-\varepsilon} \widehat{\psi}_{\mathbf{k}-\mathbf{p}, \varepsilon}^{-} \widehat{\psi}_{\mathbf{k}, \varepsilon}^{+}\right\rangle=\frac{\widetilde{\lambda}_{\infty}}{2 \pi} p\left\langle\widehat{\rho}_{\mathbf{p}, \varepsilon} \widehat{\psi}_{\mathbf{k}-\mathbf{p}, \varepsilon}^{-} \widehat{\psi}_{\mathbf{k}, \varepsilon}^{+}\right\rangle
\end{aligned}
$$


from which $(0.32),(0.33)$ are recovered. In this case the anomaly produces two different velocities in the Ward Identity, related to charge and current excitations; on the contrary, this phenomenon cannot appear in (0.101) due to Lorentz invariance, and the anomaly produces the asymmetry of the renormalization of the current and chiral current $A \neq \bar{A}$.

\subsection{Proof of the Luttinger liquid relations in non solvable models}

The conclusion of the above analysis is that the exponents of the chain model (0.34) can be written in terms of convergent expansions; such series allow to compute the indices with arbitrary precision (with explicit computation of lowest orders and a rigorous bounds of the rest), but their extreme complexity makes impossible the explicit verification of the Luttinger liquid relations (0.48) and (0.49) from them.

We have seen that the critical exponents $\eta, X_{+}, X_{-}$can be represented as power series in the variable $\lambda_{-\infty} / v_{F}$ and we can choose $\tilde{\lambda}_{\infty}(\lambda)$ and $c=v_{F}(\lambda)$ in the model (0.101) so that, by using (0.126) and (0.85)

$$
\lambda_{-\infty}(\lambda)=\widetilde{\lambda}_{-\infty}\left(\widetilde{\lambda}_{\infty}\right)
$$

As a consequence of (0.109), with this choice of $c, \widetilde{\lambda}_{\infty}$ the exponents of the model (0.101) and (0.34) are the same. In addition it is a corollary of the $\mathrm{RG}$ analysis the validity of rigorous relations between the correlations of the model (0.34) and (0.101); that is, for $|\mathbf{k}|,|\mathbf{k}+\mathbf{p}| \leq \kappa$ (in the l.h.s are correlations for the chain model (0.34) and in the r.h.s. for the model $(0.101))$

$$
\begin{aligned}
& <\widehat{\rho}_{\mathbf{p}} \widehat{a}_{\mathbf{k}+\varepsilon \mathbf{p}_{F}}^{+} \widehat{a}_{\mathbf{k}+\varepsilon \mathbf{p}+\mathbf{p}_{F}}^{-}>=\frac{Z^{(3)}}{Z^{2}}<\widehat{j}_{0, \mathbf{p}} \widehat{\psi}_{\mathbf{k}, \varepsilon}^{+} \widehat{\psi}_{\mathbf{k}+\mathbf{p}, \varepsilon}^{-}>\left(1+r_{1}\right)(0.148) \\
& <\widehat{J}_{\mathbf{p}} \widehat{a}_{\mathbf{k}+\varepsilon \mathbf{p}_{F}}^{+} \widehat{a}_{\mathbf{k}+\mathbf{p}+\mathbf{p}_{F}^{\omega}}^{-}>=\frac{\widetilde{Z}^{(3)}}{Z^{2}}<\widehat{j}_{1, \mathbf{p}} \widehat{\psi}_{\mathbf{k}, \varepsilon}^{+} \widehat{\psi}_{\mathbf{k}+\mathbf{p}, \varepsilon}^{-}>\left(1+r_{2}\right)
\end{aligned}
$$

with $\left|r_{1}\right|,\left|r_{2}\right| \leq C \kappa^{\vartheta}$ where

$$
\frac{\widetilde{Z}^{(3)}}{Z^{(3)} \sin p_{F}}=1+2 a_{1} \lambda+O\left(\lambda^{2}\right)
$$

with

$$
a_{1}=\frac{1}{2 \pi v_{F}}\left[\widehat{v}(0)-\widehat{v}\left(2 p_{F}\right)\right] .
$$

In order to prove (0.148)we note that the models (0.34) and (0.101) differ by irrelevant terms in the infrared region, and we can tune the (finite) value 
of the wave function renormalization and of the vertex or current renormalization at scale $h=0$ so that the corresponding running renormalizations are asymptotically coinciding as $h \rightarrow-\infty$. Note also that $Z^{(3)}$ and $\widetilde{Z}^{(3)}$ are different, as a consequence of the fact that in the lattice model (0.34) Lorentz symmetry is broken by the irrelevant terms; this can be explicitly checked at lowest order computing the two graphs in fig 0.8 for the lattice model (0.34).
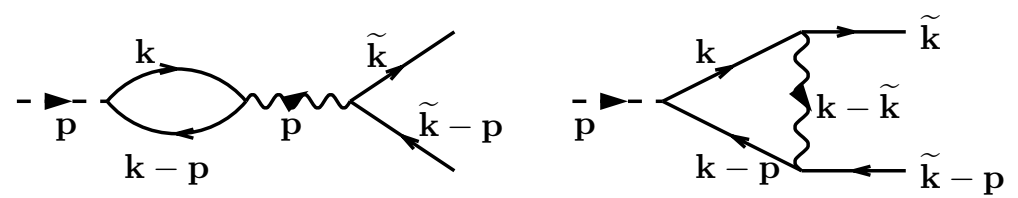

Fig. 0.8 The first order contributions to $Z^{(3)}$ and $\widetilde{Z}^{(3)}$, giving the value is $\pm a_{1}$

Similarly the current-current or the density-density renormalizations are related by the following relations

$$
\begin{aligned}
& <\widehat{\rho}_{\mathbf{p}} \widehat{\rho}_{-\mathbf{p}}>=\left[\frac{Z^{(3)}}{Z}\right]^{2}<\widehat{j}_{0, \mathbf{p}} \widehat{j}_{0,-\mathbf{p}}>+\widehat{A}_{\rho, \rho}(\mathbf{p}) \\
& <\widehat{J}_{\mathbf{p}} \widehat{J}_{-\mathbf{p}}>=\left[\frac{\widetilde{Z}^{(3)}}{Z}\right]^{2}<\widehat{j}_{1, \mathbf{p}} \widehat{j}_{i,-\mathbf{p}}>+\widehat{A}_{j, j}(\mathbf{p})
\end{aligned}
$$

with

$$
\left|A_{\rho, \rho}(\mathbf{x})\right|,\left|A_{j, j}(\mathbf{x})\right| \leq \frac{C}{|\mathbf{x}|^{2+\vartheta}}
$$

Therefore $A_{\rho, \rho}(\mathbf{p}), A_{j, j}(\mathbf{p})$ are continuous (and with a nonvanishing limit in $\mathbf{p}=0$ ), while $<\widehat{\rho}_{\mathbf{p}} \widehat{\rho}_{-\mathbf{p}}>,<\widehat{J}_{\mathbf{p}} \widehat{J}_{-\mathbf{p}}>$ are not continuous; this regularity improvement is a consequence of the fact that such terms are generated by the presence of the irrelevant terms.

With the above choice of $\widetilde{\lambda}_{\infty}$ and $c=v_{F}$ the exponents of the chain model (0.34) coincides with the exponents of (0.101), which are expressed by $(0.136)$ and $(0.139)$, so that the expoents verify Luttinger liquid relations (0.48) with

$$
K=\frac{1-\left(\widetilde{\lambda}_{\infty} / 4 \pi v_{F}\right)}{1+\left(\widetilde{\lambda}_{\infty} / 4 \pi v_{F}\right)}
$$

. and

$$
\widetilde{\lambda}_{\infty}=\lambda\left[\widehat{v}(0)-\widehat{v}\left(2 p_{F}\right)\right]+O\left(\lambda^{2}\right) \quad v_{F}=\sin p_{F}+O(\lambda)
$$


depending from all the model details. It remains to prove (0.49). Note first that the Ward Identies of the model (0.101), namely (0.129), implies the following Ward Identities for the model (0.34), for $|\mathbf{k}|,|\mathbf{k}+\mathbf{p}| \leq \kappa$, through (0.148)

$$
\begin{aligned}
& -i p_{0}<\widehat{\rho}_{\mathbf{p}} \widehat{a}_{\mathbf{k}+\varepsilon \mathbf{p}_{F}}^{+} \widehat{a}_{\mathbf{k}+\mathbf{p}+\varepsilon \mathbf{p}_{F}}^{-}>+\varepsilon p v_{F} \frac{Z^{(3)}}{\widetilde{Z}^{(3)}}<\widehat{J}_{\mathbf{p}} \widehat{a}_{\mathbf{k}+\varepsilon \mathbf{p}_{F}}^{+} \widehat{a}_{\mathbf{k}+\mathbf{p}+\varepsilon \mathbf{p}_{F}}^{-}>= \\
& \frac{Z^{(3)}}{Z} \frac{1}{(1-\tau)}\left[\left\langle\widehat{a}_{\mathbf{k}+\varepsilon \mathbf{p}_{F}}^{+} \widehat{a}_{\mathbf{k}+\varepsilon \mathbf{p}_{F}}^{-}\right\rangle-\left\langle\widehat{a}_{\mathbf{k}+\mathbf{p}+\varepsilon \mathbf{p}_{F}}^{+} \widehat{a}_{\mathbf{k}+\mathbf{p}+\varepsilon \mathbf{p}_{F}}^{-}\right\rangle\right]\left(1+O\left(\kappa^{\vartheta}\right)(0.155)\right.
\end{aligned}
$$

and

$$
\begin{aligned}
& -i p_{0}<\widehat{J}_{\mathbf{p}} \widehat{a}_{\mathbf{k}}^{+} \widehat{a}_{\mathbf{k}+\mathbf{p}}^{-}>+\varepsilon p v_{F} \frac{\widetilde{Z}^{(3)}}{Z^{(3)}}<\widehat{\rho}_{\mathbf{p}} \widehat{a}_{\mathbf{k}}^{+} \widehat{a}_{\mathbf{k}+\mathbf{p}}^{-}>= \\
& \frac{\widetilde{Z}^{(3)}}{Z} \frac{1}{(1+\tau)}\left[\left\langle\widehat{a}_{\mathbf{k}}^{+} \widehat{a}_{\mathbf{k}}^{-}\right\rangle-\left\langle\widehat{a}_{\mathbf{k}+\mathbf{p}}^{+} \widehat{a}_{\mathbf{k}+\mathbf{p}}^{-}\right\rangle\right]\left(1+O\left(\kappa^{\vartheta}\right)\right)
\end{aligned}
$$

The first Ward Identity must coincide with the exact Ward Identity found for the model $(0.34)$, obtained by the continuity equation; we see then that the finite renormalizations are not independent but are related by the following exact relations

$$
\frac{Z^{(3)}}{(1-\tau) Z}=1 \quad v_{F} \frac{Z^{(3)}}{\widetilde{Z}^{(3)}}=1
$$

from which

$$
K=1-\frac{1}{\pi v_{F}}\left[\widehat{v}(0)-\widehat{v}\left(2 p_{F}\right)\right] \lambda+O\left(\lambda^{2}\right)
$$

By (0.156) and calling $J_{\mathbf{p}}=v_{F} j_{\mathbf{p}}$ we get

$$
\begin{aligned}
& -i p_{0}<\widehat{\rho}_{\mathbf{p}} \widehat{a}_{\mathbf{k}+\varepsilon \mathbf{p}_{F}}^{+} \widehat{a}_{\mathbf{k}+\mathbf{p}+\varepsilon \mathbf{p}_{F}}^{-}>+\varepsilon p v_{F}<\widehat{j}_{\mathbf{p}} \widehat{a}_{\mathbf{k}+\varepsilon \mathbf{p}_{F}}^{+} \widehat{a}_{\mathbf{k}+\mathbf{p}+\varepsilon \mathbf{p}_{F}}^{-}>= \\
& {\left[\left\langle\widehat{a}_{\mathbf{k}+\varepsilon \mathbf{p}_{F}}^{+} \widehat{a}_{\mathbf{k}+\varepsilon \mathbf{p}_{F}}^{-}\right\rangle-\left\langle\widehat{a}_{\mathbf{k}+\mathbf{p}+\varepsilon \mathbf{p}_{F}}^{+} \widehat{a}_{\mathbf{k}+\mathbf{p}+\varepsilon \mathbf{p}_{F}}^{-}\right\rangle\right]\left(1+O\left(\kappa^{\vartheta}\right)\right)}
\end{aligned}
$$

and

$$
\begin{aligned}
& -i p_{0}<\widehat{j}_{\mathbf{p}} \widehat{a}_{\mathbf{k}}^{+} \widehat{a}_{\mathbf{k}+\mathbf{p}}^{-}>+\varepsilon p v_{F}<\widehat{\rho}_{\mathbf{p}} \widehat{a}_{\mathbf{k}}^{+} \widehat{a}_{\mathbf{k}+\mathbf{p}}^{-}>= \\
& K\left[\left\langle\widehat{a}_{\mathbf{k}}^{+} \widehat{a}_{\mathbf{k}}^{-}\right\rangle-\left\langle\widehat{a}_{\mathbf{k}+\mathbf{p}}^{+} \widehat{a}_{\mathbf{k}+\mathbf{p}}^{-}\right\rangle\right]\left(1+O\left(\kappa^{\vartheta}\right)\right)
\end{aligned}
$$

The first Ward Identity (0.158) for the chain model (0.34) was derived by the continuity equation, while the second follows from emerging chiral symmetry and cannot be derived directly by the symmetries of the hamiltonian. From (0.132) and (0.151) we get, if $D_{\varepsilon}(\mathbf{p})=-i \omega+\varepsilon v_{F} p$

$$
<\widehat{\rho}_{\mathbf{p}} \widehat{\rho}_{-\mathbf{p}}>=\left[\frac{Z^{(3)}}{Z}\right]^{2} \frac{1}{4 \pi v_{F}} \frac{1}{1-\tau^{2}}\left[\frac{D_{-}(\mathbf{p})}{D_{+}(\mathbf{p})}+\frac{D_{+}(\mathbf{p})}{D_{-}(\mathbf{p})}-2 \tau\right]+\widehat{A}_{\rho, \rho}(\mathbf{p})
$$


As we noticed $\widehat{A}_{\rho, \rho}(\mathbf{p})$ is continuous and its value at $\mathbf{p}=0$ is determined by the condition (0.43), that is $<\widehat{\rho}_{\mathbf{p}} \widehat{\rho}_{-\mathbf{p}}>_{0, p}=0$; with this value of $\widehat{A}_{\rho, \rho}(0)$ we het

$$
<\widehat{\rho}_{\mathbf{p}} \widehat{\rho}_{-\mathbf{p}}>=\left[\frac{Z^{(3)}}{Z}\right]^{2} \frac{1}{4 \pi v_{F}} \frac{1}{1-\tau^{2}} \frac{v_{F}^{2} p^{2}}{\omega^{2}+v_{F}^{2} p^{2}}+O(p)
$$

Finally, using (0.153) and (0.156) we get

$$
<\widehat{\rho}_{\mathbf{p}} \widehat{\rho}_{-\mathbf{p}}>=\frac{K}{\pi v_{F}} \frac{v_{F}^{2} p^{2}}{\omega^{2}+v_{F}^{2} p^{2}}+O(p)
$$

and the conclusion is that

$$
\kappa=\lim _{p \rightarrow 0} \lim _{\omega \rightarrow 0}<\widehat{\rho}_{\mathbf{p}} \widehat{\rho}_{-\mathbf{p}}>=\frac{K}{\pi v_{F}}
$$

In the same way we get

$$
<\widehat{J}_{\mathbf{p}} \widehat{J}_{-\mathbf{p}}>=\left[\frac{\widetilde{Z}^{(3)}}{Z}\right]^{2} \frac{1}{4 \pi v_{F}} \frac{1}{1-\tau^{2}}\left[\frac{D_{-}(\mathbf{p})}{D_{+}(\mathbf{p})}+\frac{D_{+}(\mathbf{p})}{D_{-}(\mathbf{p})}+2 \tau\right]+\widehat{A}_{j, j}(\mathbf{p})
$$

and using (0.153) and (0.156) we get

$$
D(\mathbf{p})=\frac{K v_{F}}{\pi} \frac{\omega^{2}}{\omega^{2}+v_{F}^{2} p^{2}}+O(\omega)
$$

so that

$$
D=\lim _{\omega \rightarrow 0} \lim _{p \rightarrow 0} D(\mathbf{p})=\frac{K v_{F}}{\pi}
$$

This concludes the proof of (0.48) (0.49) for the a generic non solvable chain model (0.34) and coupling not too large.

\subsection{Conclusions}

We have reviewed a recent approach to one dimensional systems of interacting fermions based on exact Renormalization Group and Constructive Quantum Field Theory methods. The exponents and several physical quantities can be written in terms of convergent expansions and several Luttinger liquid relations are rigorously established, via a combination of regularity results and emerging Ward Identites related to asymptotic symmetries. Such relations are true in the Luttinger model, whose linear dispersion relation allows an exact solution, but their validity in more 
realistic fermionic models with non linear dispersion relation (as lattice or Jellium models), even if widely accepted, was unproven except in certain particular solvable cases. The approach reviewed above proves the validity of the Luttinger liquid conjecture, in the Euclidean region and for coupling not too large (the analytic estimate of the convergence radius could be improved by computer assisted analysis, as for KAM series), for generic 1D systems solvable or not solvable; we have focused to spinless lattice fermions for definiteness, but the proof holds for continuum Jellium models or spinning Hubbard models with repulsive interaction in the non half filled band case. A similar approach has been applied also to classical bidimensional spin models like the Ashkin-Teller or the Eight vertex or any models of coupled spins with a quartic interaction [47; 43], where a proof of several of the Kadanoff relations between critical exponents [9] has been achieved. An important feature of this approach is that the irrelevant terms are fully taken into account: the analysis is performed at Euclidean times but the irrelevant terms are expected to play an even more important role at real times. Therefore, it would be important to extend such exact methods to the computation of the dynamic responses at real frequencies. 
Book Title 


\section{Bibliography}

[1] S. Tomonaga Prog. Theor. Phys. 5, 544 (1950)

[2] J.M. Luttinger J.Math. Phys. 4, 1154 (1963)

[3] W. Thirring Ann. of Phys 3, 91 (1958)

[4] K. Johnson Nuovo Cimento 20, 773-790, (1961).

[5] D. Mattis, E. Lieb. J. Math. Phys. 6, 304-312 (1965)

[6] D.M.Haldane: Phys.Rev.Lett. 45, 1358-1362, (1980)

[7] D.M.Haldane: J.Phys. C 14, 2585 (1981)

[8] Luther A., Peschel I.: Phys. Rev. B 12, 3908-3917, (1975).

[44] Kadanoff L.P.: Phys. Rev. Lett. 39, 903-905, (1977).

[10] A. W. Overhauser Phys. 1 (307) (1965)

[11] A. Theunmann Phys. Rev. B 15,4524 (1977)

[12] G.Benfatto, G.Gallavotti, V.Mastropietro Phys. Rev. B45, 10, 5468 (1991)

[13] V.Mastropietro Nuovo Cimento 109, 11 (1993)

[14] W.Metzner, C. Di Castro Phys. Rev. B 47,16107 (1993)

[15] T.D.Schultz,D.C.Mattis, E.H.Lieb. Rev. Mod. Phys36, 856 (1964)

[16] Sutherland D J.Math.Phys 11,3183-86 (1970)

[17] Baxter R.J.: Exactly solved models in statistical mechanics. Academic Press, Inc. London, (1989)

[18] Yang C.N.,Yang C.P. Phys. Rev. 147, 303-306 (1966)

[19] G. Gomez Santos Phys. Rev. B 46, 1421714218 (1992)

[20] A.A. Abrikosov, L.P. Gorkov, I.E. Dzylaloshinski Methods of Quantum Field Theory in statistical physics Prentice Hall (1963)

[21] I.E. Dzylaloshinski, A.I. Larkin Sov. phys. JETP, 38, 1, 202 (1974)

[22] J. Solyom Advances in Physics, 28, 2, 209 (1979)

[23] Wilson K.G.: Phys. Rev. B 4, 3174-3184, 1971.

[24] Polchinski,J. Nucl. Phys. B 231 , 269 (1984)

[25] Gallavotti, G. Rev. Mod. Phys. 57, 471-562 (1985).

[26] Benfatto G., Gallavotti G: Jour. Stat. Phys. 59, 541-664 (1990).

[27] Gawedzki K., Kupiainen A.: Comm.Math.Phys. 102, 1-30, 1985.

[28] Feldman J., Magnen J., Rivasseau V, Sénéor R.: Comm. Math. Phys. 103, 67-103, 1986.

[29] A. Lesniewski: Comm. Math. Phys. 108, 437-467, (1987). 
[30] Benfatto G., Gallavotti G, Procacci, A, Scoppola B: Comm. Math. Phys. 160, 93-171 (1994).

[31] Benfatto G., Mastropietro V.: Rev. Math. Phys. 13, 1323-1435, (2001).

[32] Benfatto G., Mastropietro V.: Comm. Math. Phys. 231, 1, 97-134 (2002)

[33] Benfatto G., Mastropietro V.: Comm. Math. Phys. 258, 609-655, (2005).

[34] Mastropietro V.: J. Math. Phys 48, 022302, (2007)

[35] Benfatto G., Falco P., Mastropietro V. Comm. Math. Phys. 292,2, 569-605 (2009)

[36] Benfatto G., Falco P., Mastropietro V. 'Phys.Rev.Lett 104, 075701 (2010)

[37] Benfatto G., Mastropietro V. J. Stat. Phys. 138, 6,1084 (2010)

[38] Benfatto G., Mastropietro V.. J. Stat. Phys. 142, 2,251 (2010)

[39] Mastropietro V.: Non-perturbative Renormalization. World Scientific, (2008).

[40] Caianiello E.R..: Nuovo Cimento 10, 1634, (1960).

[41] Battle G., Federbush P.: Ann.Phys. 142,95, 1982; Brydges D.: A short course on Cluster Expansions, Les Houches 1984, K. Osterwalder, R. Stora eds., North Holland Press, (1986).

[42] Mastropietro V.: J. Phys. A 40, 10349-10365, (2007).

[43] Benfatto G., Falco P, Mastropietro V: Comm. Math. Phys. 273, 67-118 (2007).

[44] Adler S. L., Bardeen W.A.: Phys. Rev. 182, 1517-1536, 1969.

[45] Georgi H., Rawls J.M.: Phys. Rev.D 3, 874-879, 1971.

[46] Gentile G., Scoppola B.: Comm. Math. Phys. 154, 135-179 (1993).

[47] Mastropietro V.: Comm. Math. Phys. 244, 595-64 (2004). 